\title{
DYNAMIC STIFFNESS METHOD FOR CIRCULAR STOCHASTIC TIMOSHENKO BEAMS: RESPONSE VARIABILITY AND RELIABILITY ANALYSES
}

\author{
S. Gupta and C. S. Manohar \\ Department of Civil Engineering, Indian Institute of Science, Bangalore 560012, \\ India.E-mail:manohar@civil.iisc.ernet.in
}

(Received 9 January 2001, and in final form 6 November 2001)

\begin{abstract}
The problem of characterizing response variability and assessing reliability of vibrating skeletal structures made up of randomly inhomogeneous, curved/straight Timoshenko beams is considered. The excitation is taken to be random in nature. A frequency-domain stochastic finite element method is developed in terms of dynamic stiffness coefficients of the constituent stochastic beam elements. The displacement fields are discretized by using frequency- and damping-dependent shape functions. Questions related to discretizing the inherently non-Gaussian random fields that characterize beam elastic, mass and damping properties are considered. Analytical methods, combined analytical and simulation-based methods, direct Monte Carlo simulations and simulation procedures that employ importance sampling strategies are brought to bear on analyzing dynamic response variability and assessment of reliability. Satisfactory performance of approximate solution procedures outlined in the study is demonstrated using limited Monte Carlo simulations.

(C) 2002 Elsevier Science Ltd. All rights reserved.
\end{abstract}

\section{INTRODUCTION}

Problems of response analysis and reliability assessment of structural dynamical systems, characterized by spatially inhomogeneous random properties, are currently receiving wide research attention $[1,2]$. In addition to the discretization of displacement and force fields, this class of problems requires discretization of structure property random fields [3-5]. This results in the replacement of continuously parametered, spatially varying random fields by a set of equivalent random variables. Consequently, the structural matrices become functions of these random variables. Subsequent solution steps are carried out in a probabilistic framework and typically involve eigenvalue analysis, uncoupling of equations of motion, forced response characterization and reliability estimation. This requires application of perturbation/Neumann series expansions or Monte Carlo simulation techniques that result in the determination of measures of response variability and structural reliability [6-8].

Recently, Manohar and Adhikari [9] and Adhikari and Manohar [10,11] adopted the dynamic stiffness matrix approach for analyzing dynamic response of skeletal structures made up of randomly inhomogeneous Euler-Bernoulli beams. These authors employed frequency-dependent shape functions to discretize both the displacement fields and the structure property random fields. These shape functions were also dependent on the mass and stiffness properties of the system. This leads to a new form of frequency-dependent weighted integrals in contrast to the weighted integral approach proposed earlier [12-15] in the context of static problems. More importantly, the proposed method eliminated the need 
for performing the difficult task of stochastic eigenvalue analysis before the dynamic response could be determined. The use of frequency-dependent shape functions ensured that the discretization scheme adapted itself to the driving frequency ranges, thereby, relieving the dependency of mesh size in relation to the excitation frequency. The studies by Manohar and Adhikari were limited to the estimation of measures of response variability and could handle only Gaussian models for structural property random fields. Subsequently, Manohar et al. [16] employed extensive Monte Carlo simulations to examine the dependence of response variability in skeletal structures on the choice of probability density function (pdf) and auto-correlation function models.

In this study, the earlier formulations of Manohar and Adhikari are extended to include the following new features:

1. Development of stochastic dynamic stiffness matrix for randomly inhomogenous circular/straight Timoshenko beams.

2. Use of frequency-dependent shape functions that are additionally dependent on damping; this enables treatment of damping characteristics in a more systematic manner.

3. The structure property random fields are modelled as being non-Gaussian. Specifically, it is assumed that the structural properties, such as mass and Young's modulus, have bounded ranges which ensure that these quantities do not assume negative values. The information available on these random fields is taken to be limited to the range, mean and covariance functions. Based on this information, a first order non-Gaussian pdf is obtained by invoking the principle of maximum entropy. This, in conjunction with the knowledge on covariance functions, is further employed to develop Nataf's models for the random fields $[17,18]$.

3. An alternative random field discretization scheme, based on weighted integral approach and optimal linear expansion (OLE) [19], is used to discretize the system property random fields. This study also clarifies a few aspects relating to discretization of non-Gaussian random fields using OLE.

5. Finally, measures on reliability are estimated by using the results on first passage failure of randomly excited systems and also by using importance sampling-based Monte Carlo simulations.

\section{RANDOMLY INHOMOGENEOUS CURVED TIMOSHENKO BEAM ELEMENT}

The field equations governing the motion of an inhomogeneous circular Timoshenko beam (Figure 1) and the set of admissible boundary conditions are determined by applying Hamilton's principle [20] and are reproduced here as follows:

$$
\begin{aligned}
& \rho(\phi) A(\phi) R \frac{\partial^{2} W}{\partial t^{2}}+c_{1}(\phi) \frac{\partial W}{\partial t}-\frac{\partial}{\partial \phi}\left[\left(\frac{\bar{k} A(\phi) G(\phi)}{R}\right) \frac{\partial W}{\partial \phi}\right]+\left(\frac{E(\phi) A(\phi)}{R}\right) W \\
& -\frac{\partial}{\partial \phi}\left[\left(\frac{\bar{k} A(\phi) G(\phi)}{R}+\frac{E(\phi) A(\phi)}{R}\right) V\right]+\frac{\partial}{\partial \phi}[(\bar{k} A(\phi) G(\phi)) \Psi]=0, \\
& \rho(\phi) A(\phi) R \frac{\partial^{2} V}{\partial t^{2}}+c_{2}(\phi) \frac{\partial V}{\partial t}-\frac{\partial}{\partial \phi}\left[\left(\frac{E(\phi) A(\phi)}{R}\right) \frac{\partial V}{\partial \phi}\right]+\frac{\partial}{\partial \phi}\left[\left(\frac{\bar{k} A(\phi) G(\phi)}{R}\right) V\right] \\
& +\frac{\partial}{\partial \phi}\left[\left(\frac{E(\phi) A(\phi)}{R}+\frac{\bar{k} A(\phi) G(\phi)}{R}\right) W\right]-\bar{k} A G(\phi) \Psi=0,
\end{aligned}
$$




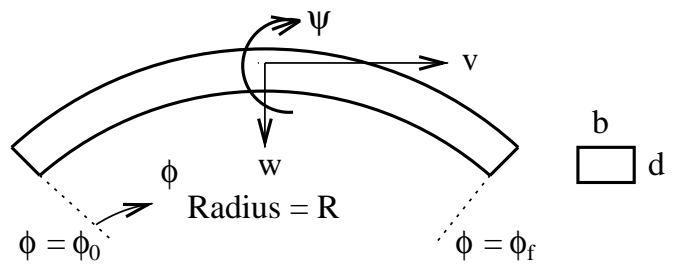

(a)

(b)

Figure 1. Inhomogeneous curved Timoshenko beam element: (a) front view, (b) cross-sectional view; $E(\phi), G(\phi)$, $\rho(\phi), b(\phi), d(\phi), c_{1}(\phi), c_{2}(\phi)$ and $c_{3}(\phi)$ are random fields.

$$
\begin{gathered}
\rho(\phi) I(\phi) R \frac{\partial^{2} \Psi}{\partial t^{2}}+c_{3}(\phi) \frac{\partial \Psi}{\partial t}-\frac{\partial}{\partial \phi}\left[\left(\frac{E(\phi) I(\phi)}{R}\right) \frac{\partial \Psi}{\partial \phi}\right]+\{\bar{k} A(\phi) G(\phi) R\} \Psi \\
-\frac{\partial}{\partial \phi}[\{\bar{k} A(\phi) G(\phi)\} W]-\{\bar{k} A(\phi) G(\phi)\} V=0 .
\end{gathered}
$$

In these equations, $E$ and $G$ are, respectively, Young's and shear modulus, $\bar{k}$ is the shape factor, $\rho$ is the mass density, $b$ and $d$ are, respectively, the breadth and depth of the cross-section, $A=b d$ is the area of the cross-section, $I=\frac{1}{12} b d^{3}$ is the moment of inertia about the axis of rotation, $R$ is the radius of curvature and $c_{1}, c_{2}$ and $c_{3}$ are the viscous damping coefficients along the radial, tangential and rotational displacements respectively. Equations (1-3) represent a set of linear partial differential equations in the spatial co-ordinate $\phi$ and time $t$, with $W, V$ and $\Psi$, respectively, denoting the radial, tangential and rotational displacements. Furthermore, the stress resultants, namely, bending moment $(\mathscr{M})$, shear force $(\mathscr{S})$ and axial force $(\mathscr{F})$ are obtained, respectively, as

$$
\begin{aligned}
& \mathscr{S}=\frac{\bar{k} A(\phi) G(\phi)}{R}\left(V+\frac{1}{R} \frac{\partial W}{\partial \phi}-R \Psi\right), \\
& \mathscr{M}=-\frac{E(\phi) I(\phi)}{R} \frac{\partial \Psi}{\partial \phi}, \quad \mathscr{F}=\frac{E(\phi) A(\phi)}{R}\left(\frac{\partial V}{\partial \phi}-W\right) .
\end{aligned}
$$

The set of admissible boundary conditions for the curved beam element at $\phi=\phi_{0}$ and $\phi=\phi_{f}$, where $\phi_{0}$ and $\phi_{f}$ are the limits of the spatial domain, are: (1) for a fixed end: $W=0$, $V=0, \Psi=0,(2)$ for a hinged end: $\mathscr{M}=0, W=0, V=0$ and (3) for a free end: $\mathscr{M}=0$, $\mathscr{S}=0$ and $\mathscr{F}=0$. In this study, the quantities $E(\phi), G(\phi), \rho(\phi), b(\phi), d(\phi), c_{1}(\phi), c_{2}(\phi)$ and $c_{3}(\phi)$ are obtained by perturbing the associated nominal values by homogenous random fields as follows:

$$
\begin{aligned}
E(\phi) & =E_{0}\left[1+\varepsilon_{1} f_{1}(\phi)\right], & G(\phi) & =G_{0}\left[1+\varepsilon_{2} f_{2}(\phi)\right], \\
b(\phi) & =b_{0}\left[1+\varepsilon_{3} f_{3}(\phi)\right], & d(\phi) & =d_{0}\left[1+\varepsilon_{4} f_{4}(\phi)\right], \\
\rho(\phi) & =\rho_{0}\left[1+\varepsilon_{5} f_{5}(\phi)\right], & & c_{1}(\phi)=c_{1_{0}}\left[1+\varepsilon_{6} f_{6}(\phi)\right], \\
c_{2}(\phi) & =c_{2_{0}}\left[1+\varepsilon_{7} f_{7}(\phi)\right], & & c_{3}(\phi)=c_{3_{0}}\left[1+\varepsilon_{8} f_{8}(\phi)\right] .
\end{aligned}
$$


In these equations, the subscript 0 indicates the nominal values, $0<\varepsilon_{k} \ll 1(k=1, \ldots, 8)$ are deterministic constants denoting the strength of the randomness and $f_{k}(\phi)(k=1, \ldots, 8)$ are assumed to be mutually independent, homogeneous random fields with mean $\mu_{k}$ and covariance $\mathscr{R}_{k k}(\tau)=\left\langle\left(f_{k}(\phi)-\mu_{k}\right)\left(f_{k}(\phi+\tau)-\mu_{k}\right)\right\rangle$. Here $\langle\cdot\rangle$ denotes the mathematical expectation operator. The following additional restrictions are taken to apply on the random fields: (1) the random fields $f_{k}(\phi)(k=1, \ldots, 8)$ are mean square bounded, (2) $f_{k}(\phi)$ $(k=1, \ldots, 4)$ are twice differentiable in the mean square sense; this requires that $\partial^{4} \mathscr{R}_{k k}\left(\phi_{1}, \phi_{2}\right) / \partial \phi_{1}^{2} \partial \phi_{2}^{2}$ must exist for all $\phi_{1}$ and $\phi_{2}$ in the interval $\phi_{0}$ and $\phi_{f}$ and (3) for a specified deterministic function $g(\phi)$, which is bounded and continuous in $\left(\phi_{0}, \phi_{f}\right)$, integrals of the type $\int_{\phi_{0}}^{\phi_{f}} g(\phi) f_{k}(\phi) \mathrm{d} \phi$ exist in a mean square sense; this requires that $\int_{\phi_{0}}^{\phi_{s}} \int_{\phi_{0}}^{\phi_{s}}\left|g\left(\phi_{1}\right) g\left(\phi_{2}\right) \mathscr{R}_{k k}\left(\phi_{1}, \phi_{2}\right)\right| \mathrm{d} \phi_{1} \mathrm{~d} \phi_{2}<\infty(k=1, \ldots, 8)$. The first two conditions ensure that the sample realizations of the beam have sufficiently smooth behaviour so that the various stress resultants and boundary conditions (such as those at the free edge) are satisfactorily described. The third condition is required for the development of the procedure used in this study. It may be noted that these conditions impose restrictions essentially on the covariance model of the random fields. Furthermore, since the quantities on the right hand-side of equation (7) denote strictly positive physical parameters, $f_{k}(\phi)$ $(k=1, \ldots, 8)$, are required to satisfy the condition $P\left[1+\varepsilon_{k} f_{k}(\phi)>0\right]=1, P[\cdot]$ denoting the probability measure, thus imposing a restriction on the pdf model of the random fields $f_{k}(\phi)$. It may be noted that, although $f_{k}(\phi)(k=1, \ldots, 8)$ are taken to be mutually independent, these models still ensure that the quantities $E I(\phi), G A(\phi)$ and $\rho A(\phi)$ remain mutually dependent as might be expected. It is to be noted that the nominal cross-section of the beam is taken to be rectangular. By virtue of stochastic perturbations impressed on $b(\phi)$ and $d(\phi)$, the sample realization of beam cross-section depart from strict rectangular shape. On account of this, the beam displacements $W, V$ and $\Psi$ can be expected to get coupled to the twisting of the beam. This secondary coupling effect, however, is not considered in this study.

In order to postulate models for probability distribution, it is assumed that the information on $f_{k}(\phi)$ is limited to ranges $\left(a_{k}, b_{k}\right)$ on $f_{k}(\phi)$ such that $P\left[a_{k}<f_{k}(\phi)<b_{k}\right]=1$, for all $\phi, a_{k}>-\infty, a_{k}<b_{k}<\infty$, mean $\mu_{k}$ and covariance $R_{k k}(\tau)$. This would mean that a complete knowledge on pdf of $f_{k}(\phi)$ is assumed to be lacking. This assumption is believed to be realistic, given the current state of knowledge in modelling of structural uncertainties [2]. It must be noted here that the limited available information on $f_{k}(\phi)$ is inadequate for carrying out the response analysis, especially, using simulation methods. To overcome this problem, it is proposed that a model for the first order pdf of $f_{k}(\phi)$ be constructed by invoking the principle of maximum entropy [21]. This involves finding $p_{f_{k}}(\zeta)$ that maximize entropy $\mathscr{H}$ given by

$$
\mathscr{H}=-\int_{a_{k}}^{b_{k}} p_{f_{k}}(\zeta) \log p_{f_{k}}(\zeta) \mathrm{d} \zeta
$$

subject to the constraints

$$
\begin{gathered}
\int_{a_{k}}^{b_{k}} p_{f_{k}}(\zeta) \mathrm{d} \zeta=1, \quad \int_{a_{k}}^{b_{k}} \zeta p_{f_{k}}(\zeta) \mathrm{d} \zeta=\mu_{k}, \\
\int_{a_{k}}^{b_{k}}\left(\zeta-\mu_{k}\right)^{2} p_{f_{k}}(\zeta) \mathrm{d} \zeta=\sigma_{k}^{2}=\mathscr{R}_{k k}(0) .
\end{gathered}
$$


Using variational calculus, it can be shown that the resulting optimal pdf has the form

$$
p_{f_{k}}(\zeta)=\lambda_{1_{k}} \exp \left[-\lambda_{2_{k}} \zeta-\lambda_{3_{k}}\left(\zeta-\mu_{k}\right)^{2}\right], \quad a_{k}<\zeta<b_{k},
$$

where the constants $\lambda_{1_{k}}, \lambda_{2_{k}}$ and $\lambda_{3_{k}}$ are selected so that the constraint equations (9-11) are satisfied. Furthermore, this model for the first order pdf is combined with the known covariance function $\mathscr{R}\left(\zeta_{1}, \zeta_{2}\right)$ to derive Nataf's model for the higher order pdfs following the procedure outlined in reference [18]. This leads to the joint pdf

$$
p_{f_{1} \ldots f_{8}}\left(\zeta_{1}, \ldots, \zeta_{8}\right)=h_{V_{1}, \ldots, V_{8}}\left(\eta_{1}, \ldots, \eta_{8}\right) \frac{p_{f_{1}}\left(\zeta_{1}\right) \ldots p_{f_{8}}\left(\zeta_{8}\right)}{h_{V_{1}}\left(\eta_{1}\right) \ldots h_{V_{8}}\left(\eta_{8}\right)}
$$

where $V_{1}, \ldots, V_{8}$ are standard normal variates obtained by the memoryless transformations on $f_{1}, \ldots, f_{8}$ given by

$$
\zeta_{k}=H_{V_{k}}^{-1}\left[P_{f_{k}}\left(\zeta_{k}\right)\right], \quad k=1, \ldots, 8 .
$$

Here $P_{f_{k}}\left(\zeta_{k}\right)(k=1, \ldots, 8)$ are the probability distribution functions (PDF) of $f_{k}, H_{V_{k}}\left(\eta_{k}\right)$ are the PDF of the marginal normal pdf $h_{V_{k}}\left(\eta_{k}\right)$ and $h_{V_{1} \ldots V_{8}}\left(\eta_{1}, \ldots, \eta_{8}\right)$ is the joint normal pdf with zero mean, unit standard deviation and unknown correlation coefficient matrix $\mathscr{Y}$. These correlation coefficients $\mathscr{Y}_{k j}$ are in turn expressed in terms of the correlation coefficients $\mathscr{R}_{k j}$ through the integral equations

$$
\mathscr{R}_{k j}=\int_{a_{k}}^{b_{k}} \int_{a_{j}}^{b_{j}}\left(\frac{\eta_{k}-\mu_{k}}{\sigma_{k}}\right)\left(\frac{\eta_{j}-\mu_{j}}{\sigma_{j}}\right) h_{V_{k} V_{j}}\left(\eta_{k}, \eta_{j} ; \mathscr{Y}\right) \mathrm{d} \eta_{k} \mathrm{~d} \eta_{j}, \quad k, j=1, \ldots, 8 .
$$

These equations are solved iteratively to obtain $\mathscr{Y}_{k j}$.

\section{FORMULATION OF DYNAMIC STIFFNESS MATRIX}

Figure 2 shows a circular curved Timoshenko beam element in which harmonic displacements $\delta_{k} \exp [\mathrm{i} \omega t](k=1, \ldots, 6)$ coexist with harmonic forces $F_{k} \exp [\mathrm{i} \omega t]$

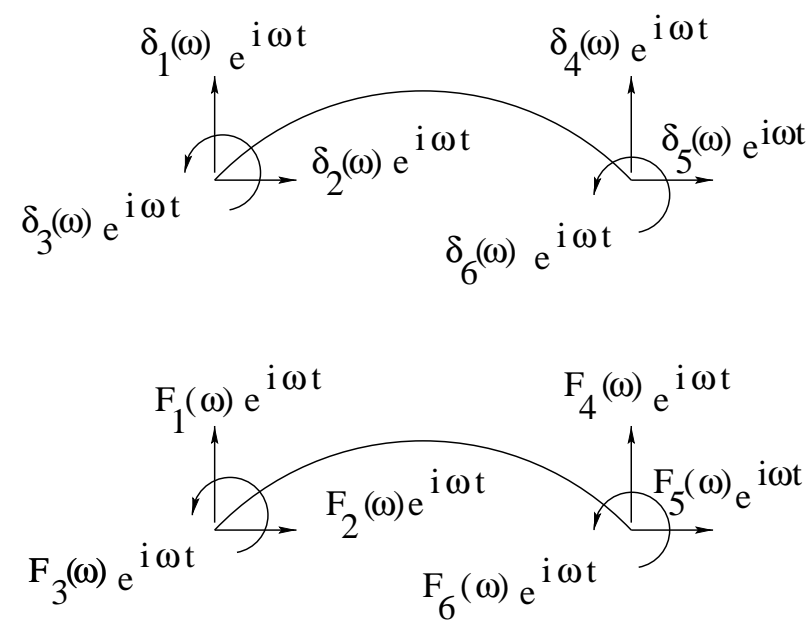

Figure 2. Displacement and force boundary conditions for formulation of the dynamic stiffness matrix for curved Timoshenko beam element. 
$(k=1, \ldots, 6)$ where $i=\sqrt{-1}$ and $\omega$ is the driving frequency. The dynamic stiffness matrix $\mathbf{D}(\omega)$ for the beam element relates these displacements $\delta(\omega)$ and forces $\mathbf{F}(\omega)$ through the equation

$$
\mathbf{D}(\omega) \delta(\omega)=\mathbf{F}(\omega)
$$

Clearly, for a damped beam element with stochastic inhomogeneities in the beam properties, the dynamic stiffness coefficients $D_{k j}(\omega)$, for a fixed $\omega$, would be complex valued random variables. To determine these coefficients, the displacements are represented as

$$
\begin{gathered}
W(\phi, t)=w(\phi) \exp [\mathrm{i} \omega t], \quad \Psi(\phi, t)=\psi(\phi) \exp [\mathrm{i} \omega t], \\
V(\phi, t)=v(\phi) \exp [\mathrm{i} \omega t] .
\end{gathered}
$$

This leads to the elimination of time dependence in equations (1-3) and the equations get simplified to ordinary differential equations of the form

$$
\begin{aligned}
& -\omega^{2} \rho(\phi) A(\phi) R w+\mathrm{i} \omega c_{1}(\phi) w-\left(\frac{\bar{k} A(\phi) G(\phi)}{R}\right) \frac{\mathrm{d}^{2} w}{\mathrm{~d} \phi^{2}}+\left(\frac{E(\phi) A(\phi)}{R}\right) w \\
& -\left(\frac{\bar{k} A(\phi) G(\phi)}{R}+\frac{E(\phi) A(\phi)}{R}\right) \frac{\mathrm{d} v}{\mathrm{~d} \phi}+\bar{k} A(\phi) G(\phi) \frac{\mathrm{d} \psi(\phi)}{\mathrm{d} \phi}=0 \\
& -\omega^{2} \rho(\phi) A(\phi) R v+\mathrm{i} \omega c_{2}(\phi) v-\left(\frac{E(\phi) A(\phi)}{R}\right) \frac{\mathrm{d}^{2} v}{\mathrm{~d} \phi^{2}}+\left(\frac{\bar{k} A(\phi) G(\phi)}{R}\right) v \\
& +\left(\frac{E(\phi) A(\phi)}{R}+\frac{\bar{k} A(\phi) G(\phi)}{R}\right) \frac{\mathrm{d} w}{\mathrm{~d} \phi}-\bar{k} A(\phi) G(\phi) \psi=0 \\
& -\omega^{2} \rho(\phi) I(\phi) R \psi+\mathrm{i} \omega c_{3}(\phi) \psi-\left(\frac{E(\phi) I(\phi)}{R}\right) \frac{\mathrm{d}^{2} \psi}{\mathrm{d} \phi^{2}}+\bar{k} A(\phi) G(\phi) R \psi \\
& -\bar{k} A(\phi) G(\phi) \frac{\mathrm{d} w}{\mathrm{~d} \phi}-\bar{k} A(\phi) G(\phi) v=0 .
\end{aligned}
$$

It may be noted that the coefficients of these equations are complex valued and are also random in nature. The determination of $\mathbf{D}(\omega)$ requires the solution of equations (20-22) under two sets of boundary conditions given by

$$
\begin{aligned}
& w\left(\phi_{0}\right)=\delta_{1}, \quad v\left(\phi_{0}\right)=\delta_{2}, \quad \psi\left(\phi_{0}\right)=\delta_{3}, \\
& w\left(\phi_{f}\right)=\delta_{4}, \quad v\left(\phi_{f}\right)=\delta_{5}, \quad \psi\left(\phi_{f}\right)=\delta_{6}
\end{aligned}
$$

and

$$
\begin{gathered}
{\left[\frac{\bar{k} A(\phi) G(\phi)}{R}\left(v+\frac{1}{R} \frac{d w}{\mathrm{~d} \phi}-R \psi\right)\right]\left(\phi_{0}\right)=F_{1}, \quad-\left[\frac{E(\phi) I(\phi)}{R} \frac{\mathrm{d} \psi}{\mathrm{d} \phi}\right]\left(\phi_{0}\right)=F_{2},} \\
{\left[\frac{E(\phi) A(\phi)}{R}\left(\frac{\mathrm{d} v}{\mathrm{~d} \phi}-w\right)\right]\left(\phi_{0}\right)=F_{3}, \quad\left[\frac{\bar{k} A(\phi) G(\phi)}{R}\left(v+\frac{1}{R} \frac{\mathrm{d} w}{\mathrm{~d} \phi}-R \psi\right)\right]\left(\phi_{f}\right)=F_{4},} \\
-\left[\frac{E(\phi) I(\phi)}{R} \frac{\mathrm{d} \psi}{\mathrm{d} \phi}\right]\left(\phi_{f}\right)=F_{5}, \quad\left[\frac{E(\phi) A(\phi)}{R}\left(\frac{\mathrm{d} v}{\mathrm{~d} \phi}-w\right)\right]\left(\phi_{f}\right)=F_{6} .
\end{gathered}
$$


Thus, equations (20-22), together with the boundary conditions in equations (23) and (24), constitute a set of stochastic boundary value problems. An exact solution to this problem is currently not feasible. To proceed further, it is essential to either employ an approximate procedure or resort to Monte Carlo simulations. In this study, Galerkin's method is used to seek approximate solutions and these are validated using Monte Carlo simulations.

\subsection{DISCRETIZATION OF DISPLACEMENT FIELDS}

The first step in the implementation of Galerkin's method is to represent the displacement fields as

$$
\begin{gathered}
w(\phi, \omega)=\sum_{k=1}^{6} Z_{k}(\omega) N_{k}(\phi, \omega), \quad v(\phi, \omega)=\sum_{k=1}^{6} Z_{k}(\omega) P_{k}(\phi, \omega), \\
\psi(\phi, \omega)=\sum_{k=1}^{6} Z_{k}(\omega) Q_{k}(\phi, \omega),
\end{gathered}
$$

where $Z_{k}(\omega)$ are the generalized coordinates and $N_{k}(\phi, \omega), P_{k}(\phi, \omega)$ and $Q_{k}(\phi, \omega)$ $(k=1, \ldots, 6)$ are the displacement shape functions. In this study, the shape functions are derived by solving the field equations (20-22) with the beam properties taken to be independent of $\phi$ and equal to their nominal values, i.e., $E_{0} I_{0}, G_{0} A_{0}, \rho_{0} A_{0}, c_{1_{0}}, c_{2_{0}}$ and $c_{3_{0}}$. The uncoupling of the field equations [22] and the corresponding formulation of the shape functions is described in Appendix A. The shape functions derived in this manner are functions of frequency and damping, and are thus complex valued. A distinct feature of these shape functions is that the spatial variations of these functions adapt themselves to the frequency of harmonic excitations and possess the well-known property: $N_{j}\left(\phi_{k}\right)=\delta_{j k}$, $P_{j}\left(\phi_{k}\right)=\delta_{j k}, Q_{j}\left(\phi_{k}\right)=\delta_{j k}$, where $\delta_{j k}$ is the Kronecker delta function. The plot of $\left|P_{2}(\phi, \omega)\right|$ is illustrated in Figure 3. It can be seen from this figure that at zero excitation frequency, the shape function resembles the corresponding static shape function.

\subsection{DISCRETIZATION OF RANDOM FIELDS}

One of the key steps in the application of finite element method to problems involving stochastic inhomogeneities is the discretization of the system property random fields. In the present study, two alternative schemes are considered for this purpose.

\subsubsection{Weighted integral approach}

In this method, the random fields are discretized implicitly using the same shape functions that have been used in discretizing the displacement fields (section 3.1). This typically results in integrals of the form

$$
\mathscr{W}_{k l}(\omega)=\int_{\phi_{0}}^{\phi_{f}} \mathscr{G}\left[f_{1}(\phi), \ldots, f_{8}(\phi)\right] N_{k}(\phi, \omega) N_{l}(\phi, \omega) \mathrm{d} \phi,
$$

where the function $\mathscr{G}\left[f_{1}(\phi), \ldots, f_{8}(\phi)\right]$ is obtained by the combination of various system random fields required for representing a particular quantity, e.g., $E A(\phi)=E(\phi) b(\phi) d(\phi)$. Clearly, for a fixed $\omega, \mathscr{W}_{k l}(\omega)$ is a random variable. Furthermore, in the present study, since the shape functions are complex valued, the weighted integrals, for a fixed $\omega$, in turn, 


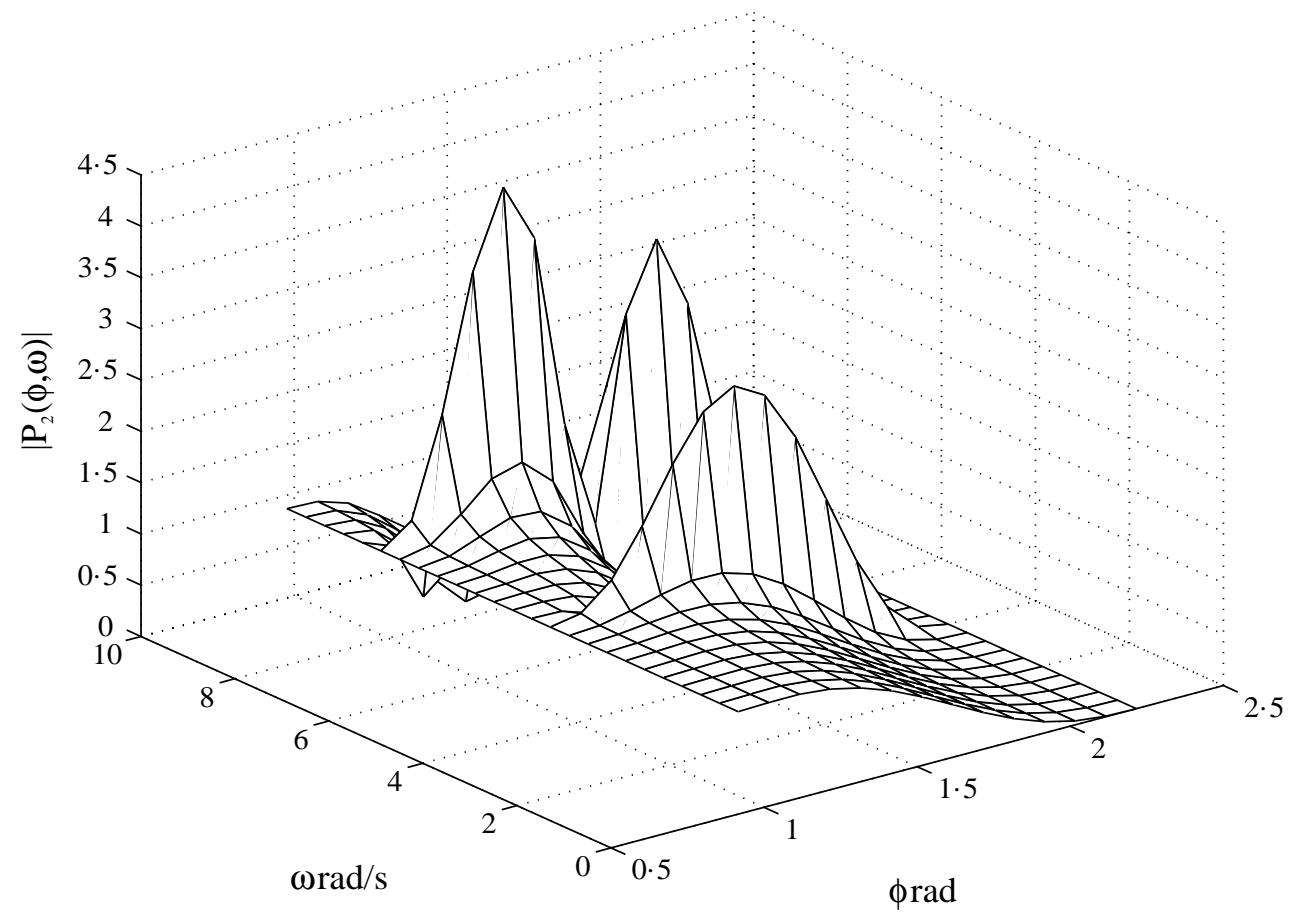

Figure 3. Shape function $P_{2}(\phi, \omega)$ with $\phi_{0}=0.9153 \mathrm{rad}$ and $\phi_{f}=2.2263 \mathrm{rad}$.

become complex valued random variables. It may be noted that, for static applications, the weighted integrals are real valued with dependency on frequency being of no relevance $[14,15]$. If $\mathscr{G}\left[f_{1}(\phi), \ldots, f_{8}(\phi)\right]$ is a Gaussian field, it follows that $\mathscr{W}_{k l}(\omega)$ is a Gaussian random variable. However, Gaussian models for strictly positive quantities such as density and elastic rigidities are inappropriate, especially, if measures on structural reliability are to be estimated. On the other hand, if $\mathscr{G}\left[f_{1}(\phi), \ldots, f_{8}(\phi)\right]$ is non-Gaussian; in general, it is not possible to obtain the probability distribution of $\mathscr{W}_{k l}(\omega)$ [23]. However, the moments of the weighted integrals can be obtained in terms of moments of $\mathscr{G}\left[f_{1}(\phi), \ldots, f_{8}(\phi)\right][24]$.

\subsubsection{Optimal linear expansion}

In this method, the choice of the shape functions used for discretizing the structure property random fields are divorced from any considerations on discretization of displacement fields. Here, a random field is represented by

$$
\tilde{f}(\phi)=\sum_{k=1}^{n} S_{k}(\phi) f\left(\phi_{k}\right), \quad \phi_{0} \leqslant \phi \leqslant \phi_{f},
$$

where $S_{k}(\phi)$ are deterministic functions, $n$ is the number of nodal points and $f\left(\phi_{k}\right)$ are random variables. Following Li and Der Kiureghian [19], $S_{k}(\phi)$ are selected such that the variance of error of discretization, given by

$$
\left\langle\varepsilon_{0}^{2}\right\rangle=\left\langle\left\{f(\phi)-\sum_{k=1}^{n} S_{k}(\phi) f\left(\phi_{k}\right)\right\}^{2}\right\rangle
$$


is minimized, subject to the constraint that $\left\langle\varepsilon_{0}\right\rangle=0$. This leads to the solution

$$
\mathbf{S}(\phi)=\mathscr{B}^{-1} \mathscr{V},
$$

where $\mathscr{V}$ is an $n \times 1$ vector of expectations $\left\langle f(x) f\left(\phi_{k}\right)\right\rangle,(k=1, \ldots, n), \mathscr{B}$ is the covariance matrix of $\mathbf{f}$, assumed to be non-singular and $\mathbf{f}$ is an $n \times 1$ vector of random variables $f\left(\phi_{k}\right)$ $(k=1, \ldots, n)$. Thus, $S_{k}(\phi)$ are dependent on the covariance of $f(\phi)$. It is of significance to note that the shape functions $S_{k}(\phi)$, so derived, also satisfy the relation $S_{k}\left(\phi_{l}\right)=\delta_{k l}$, although this condition is not explicitly imposed as a requirement in deriving $S_{k}(\phi)$. To illustrate this, for an expansion with $n$ terms, the equation for the shape functions are written in matrix form

$$
\left[\begin{array}{cccccc}
\mathscr{B}_{11} & \mathscr{B}_{12} & \cdot & . & . & \mathscr{B}_{1 n} \\
\mathscr{B}_{12} & \mathscr{B}_{22} & \cdot & . & \cdot & \mathscr{B}_{2 n} \\
\cdot & \cdot & \cdot & \cdot & \cdot & \cdot \\
\cdot & \cdot & \cdot & \cdot & \cdot & \cdot \\
\mathscr{B}_{1 n} & \mathscr{B}_{2 n} & \cdot & \cdot & \cdot & \mathscr{B}_{n n}
\end{array}\right]\left\{\begin{array}{c}
S_{1}(\phi) \\
S_{2}(\phi) \\
\cdot \\
\cdot \\
S_{n}(\phi)
\end{array}\right\}=\left\{\begin{array}{c}
\left\langle f(\phi) f\left(\phi_{1}\right)\right\rangle \\
\left\langle f(\phi) f\left(\phi_{2}\right)\right\rangle \\
\cdot \\
\cdot \\
\left\langle f(\phi) f\left(\phi_{n}\right)\right\rangle
\end{array}\right\},
$$

where $\mathscr{B}_{j k}=\left\langle f\left(\phi_{j}\right) f\left(\phi_{k}\right)\right\rangle$. Substituting $\phi=\phi_{l}$ into equation (32) leads to

$$
\left[\begin{array}{cccccc}
\mathscr{B}_{11} & \mathscr{B}_{12} & \cdot & \mathscr{B}_{i l} & \cdot & \mathscr{B}_{1 n} \\
\mathscr{B}_{12} & \mathscr{B}_{22} & \cdot & \mathscr{B}_{2 l} & \cdot & \mathscr{B}_{2 n} \\
\cdot & \cdot & \cdot & \cdot & \cdot & \cdot \\
\mathscr{B}_{1 l} & \mathscr{B}_{2 l} & \cdot & \mathscr{B}_{l l} & \cdot & \mathscr{B}_{l n} \\
\cdot & \cdot & \cdot & \cdot & \cdot & \cdot \\
\mathscr{B}_{1 n} & \mathscr{B}_{2 n} & \cdot & \mathscr{B}_{l n} & \cdot & \mathscr{B}_{n n}
\end{array}\right]\left\{\begin{array}{c}
S_{1}\left(\phi_{l}\right) \\
S_{2}\left(\phi_{l}\right) \\
\cdot \\
S_{l}\left(\phi_{l}\right) \\
\cdot \\
S_{n}\left(\phi_{l}\right)
\end{array}\right\}=\left\{\begin{array}{c}
\left\langle f\left(\phi_{l}\right) f\left(\phi_{1}\right)\right\rangle \\
\left\langle f\left(\phi_{l}\right) f\left(\phi_{2}\right)\right\rangle \\
\cdot \\
\left\langle f\left(\phi_{l}\right) f\left(\phi_{l}\right)\right\rangle \\
\cdot \\
\left\langle f\left(\phi_{l}\right) f\left(\phi_{n}\right)\right\rangle
\end{array}\right\}=\left\{\begin{array}{c}
\mathscr{B}_{1 l} \\
\mathscr{B}_{2 l} \\
\cdot \\
\mathscr{B}_{l l} \\
\cdot \\
\mathscr{B}_{n l}
\end{array}\right\} .
$$

Noting that $\mathscr{B}_{j k}=\mathscr{B}_{k j}$, it may be verified by direct substitution that, $S_{k}\left(\phi_{l}\right)=\delta_{k l}$ $(k=1, \ldots, n)$ is a solution of equation (33). Furthermore, since the rank of the coefficient matrix in the above equation is $n$, the solution $S_{k}\left(\phi_{l}\right)=\delta_{k l}$ is the only solution. This property is illustrated in Figure 4 where the first six shape functions are obtained for a random field with covariance function of the form $R_{f f}(\tau)=\exp \left[-\alpha \tau^{2}\right]$, with $\alpha=7 \cdot 3$, which is used in the examples considered later in the paper. The property that $S_{k}\left(\phi_{l}\right)=\delta_{k l}$ is clearly evidenced in this figure. As a consequence of this property, it follows that the first order pdf of $\tilde{f}(\phi)$ matches exactly with the corresponding pdf of $f(\phi)$ for $\phi=\phi_{l}(l=1, \ldots, n)$. This implies that the mean square error $\left\langle\varepsilon_{0}^{2}\right\rangle$ becomes zero at $\phi=\phi_{l}(l=1, \ldots, n)$. The choice of $n$ is made by requiring that the global error $\int_{\phi_{0}}^{\phi_{s}}\left\langle\varepsilon_{0}^{2}\right\rangle \mathrm{d} \phi$ remains less than the prescribed limit. Figure 5 shows the PDF of $\tilde{f}(\phi)$ at $\phi=\phi_{1}$ obtained using Monte Carlo simulations with 500 number of samples. The plot is displayed on a normal probability paper. For the purpose of comparison, the PDF of a normal variate with the same mean and standard deviation as that of $\tilde{f}\left(\phi_{1}\right)$ is also shown in this graph. The non-Gaussian feature of $\tilde{f}\left(\phi_{1}\right)$ is clearly discernible from this figure. A similar plot for a point $\phi$ that does not coincide with any of $\phi=\phi_{l}(l=1, \ldots, n)$ is shown in Figure 6. Here, $\tilde{f}(\phi)$ is obtained as a weighted sum of $n$ non-Gaussian random variables. Notwithstanding this summation, the PDF of $\tilde{f}(\phi)$ is observed to remain non-Gaussian. Some of these features appears to have not been appreciated in the earlier work of Li and Der Kiureghian [19]. 


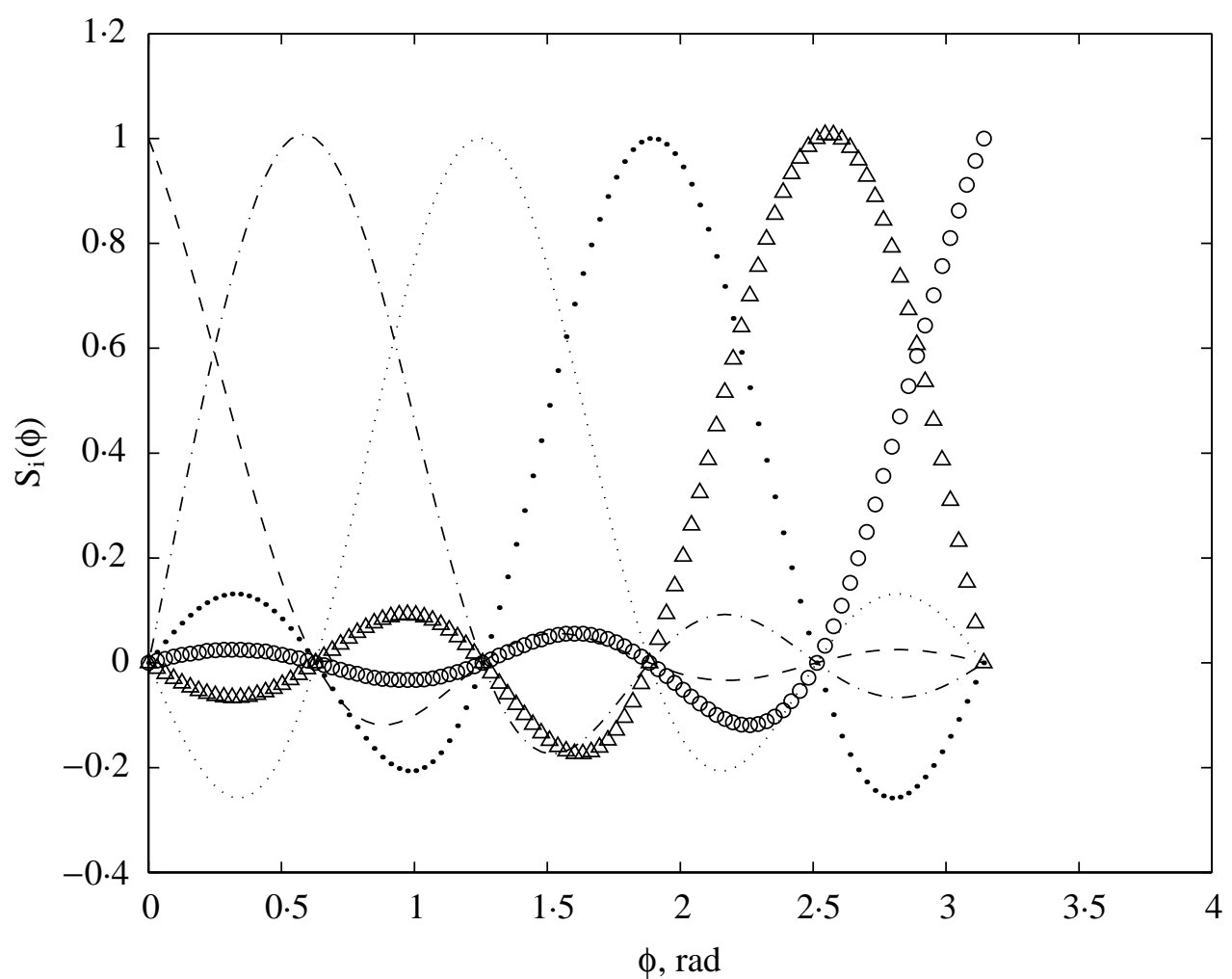

Figure 4. Shape functions $S_{i}(\phi)(i=1, \ldots, n)$ used in OLE with $\phi_{0}=0 \mathrm{rad}$ and $\phi_{f}=\pi \operatorname{rad}:---, S_{1}(\phi)$; $\cdot-\cdot \cdot-\cdot, S_{2}(\phi) ; \cdots, S_{3}(\phi) ; \bullet, S_{4}(\phi) ; \triangle, S_{5}(\phi) ; \bigcirc, S_{6}(\phi)$.

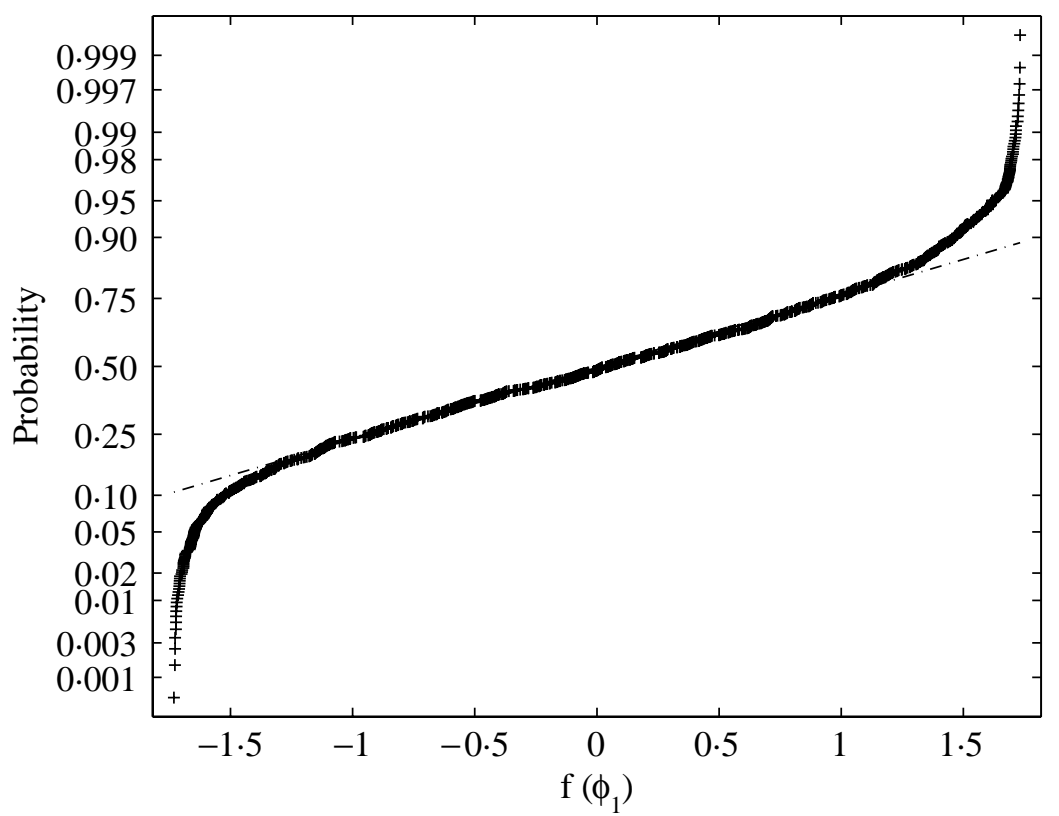

Figure 5. Illustration of non-Gaussian features of $\tilde{f}(\phi)$ in normal probability paper: ++ , probability distribution of $f\left(\phi_{1}\right)$ (node 1): $\cdot \cdot \cdot \cdot \cdot \cdot \cdot \cdot-$, corresponding Gaussian fit. 


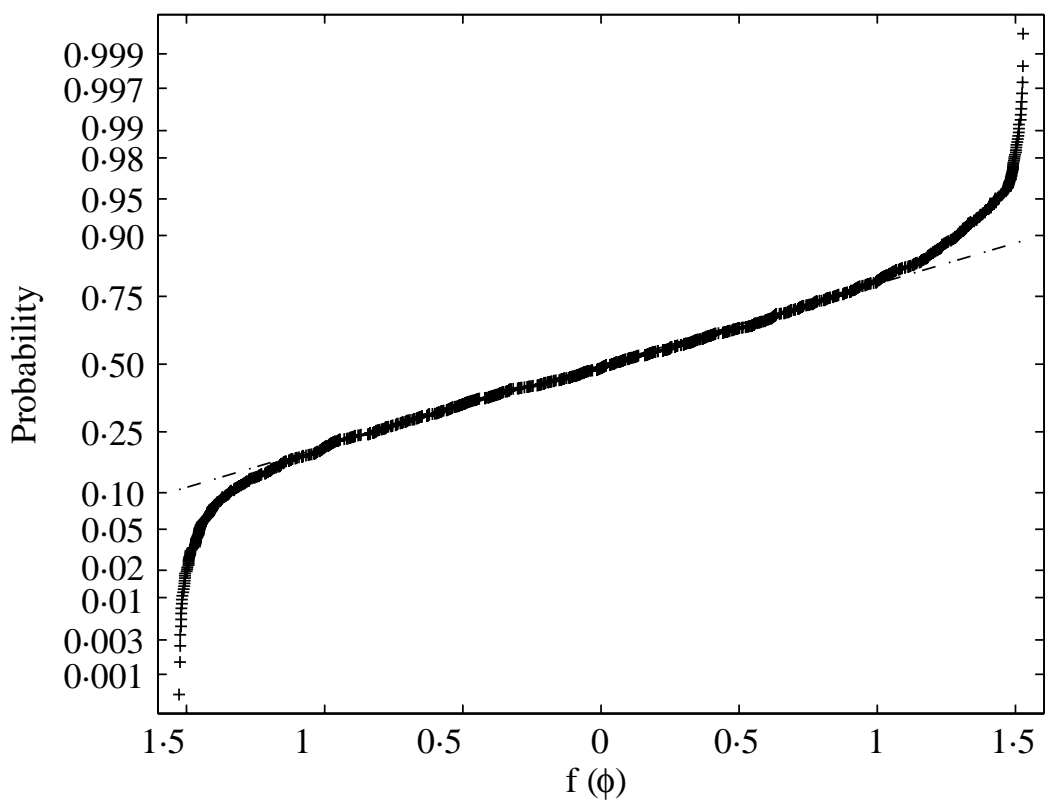

Figure 6. Illustration of non-Gaussian features of $\tilde{f}(\phi)$ in normal probability paper: + + , probability distribution of $\tilde{f}(\phi)$ at the midpoint of the section between nodes 1 and $2 ;++$, probability distribution of $\tilde{f}(\phi) ; \cdot \cdot \cdot-\cdot-\cdot-$, corresponding Gaussian fit.

\subsection{ELEMENTS OF THE STOCHASTIC DYNAMIC STIFFNESS MATRIX}

In conjunction with the displacement shape functions and the representation of the displacement fields as in equations (17-19), the expressions for the total beam kinetic energy $\mathscr{T}$ and strain energy $\mathscr{U}$ can be formulated. It can be shown that the kinetic energy is given by

$$
\mathscr{T}=\frac{1}{2} \sum_{i=1}^{6} \sum_{j=1}^{6} \dot{z}_{i}(t) \dot{z}_{j}(t) \chi_{i j}(\omega)
$$

where

$$
\chi_{i j}=\chi_{i j}^{A}+\chi_{i j}^{B}+\chi_{i j}^{C}
$$

and

$$
\begin{aligned}
& \chi_{i j}^{A}=\int_{\phi_{0}}^{\phi_{f}} R \rho(\phi) A(\phi) N_{i}(\phi, \omega) N_{j}(\phi, \omega) \mathrm{d} \phi, \\
& \chi_{i j}^{B}=\int_{\phi_{0}}^{\phi_{f}} R \rho(\phi) A(\phi) P_{i}(\phi, \omega) P_{j}(\phi, \omega) \mathrm{d} \phi, \\
& \chi_{i j}^{C}=\int_{\phi_{0}}^{\phi_{f}} R \rho(\phi) I(\phi) Q_{i}(\phi, \omega) Q_{j}(\phi, \omega) \mathrm{d} \phi .
\end{aligned}
$$

Similarly, the strain energy is given by

$$
\mathscr{U}=\frac{1}{2} \sum_{i=1}^{6} \sum_{j=1}^{6} z_{i}(t) z_{j}(t) \kappa_{i j}(\omega),
$$


where

$$
\begin{aligned}
& \kappa_{i j}=\kappa_{i j}^{1}+\kappa_{i j}^{2}+\kappa_{i j}^{3}, \quad \kappa_{\mathrm{ij}}^{2}=K_{i j}^{a}-K_{i j}^{b}-K_{i j}^{c}+K_{i j}^{d}, \\
& \kappa_{i j}^{3}=S_{i j}^{p}+S_{i j}^{q}+S_{i j}^{r}+S_{i j}^{s}-R\left(S_{i j}^{t}+S_{i j}^{u}+S_{i j}^{v}+S_{i j}^{w}\right)+R^{2} S_{i j}^{x} \\
& \kappa_{i j}^{1}=\int_{\phi_{0}}^{\phi_{f}} \frac{E I(\phi)}{R} Q_{i}^{\prime}(\phi, \omega) Q_{j}^{\prime}(\phi, \omega) \mathrm{d} \phi, \\
& K_{i j}^{a}=\int_{\phi_{0}}^{\phi_{f}} \frac{E A(\phi)}{R} P_{i}^{\prime}(\phi, \omega) P_{j}^{\prime}(\phi, \omega) \mathrm{d} \phi, \\
& K_{i j}^{b}=\int_{\phi_{0}}^{\phi_{f}} \frac{E A(\phi)}{R} N_{i}(\phi, \omega) P_{j}^{\prime}(\phi, \omega) \mathrm{d} \phi \\
& K_{i j}^{c}=\int_{\phi_{0}}^{\phi_{f}} \frac{E A(\phi)}{R} P_{i}^{\prime}(\phi, \omega) N_{j}(\phi, \omega) \mathrm{d} \phi, \\
& K_{i j}^{d}=\int_{\phi_{0}}^{\phi_{f}} \frac{E A(\phi)}{R} N_{i}(\phi, \omega) N_{j}(\phi, \omega) \mathrm{d} \phi, \\
& S_{i j}^{p}=\int_{\phi_{0}}^{\phi_{f}} \frac{k A G(\phi)}{R} P_{i}(\phi, \omega) P_{j}(\phi, \omega) \mathrm{d} \phi, \\
& S_{i j}^{q}=\int_{\phi_{0}}^{\phi_{f}} \frac{k A G(\phi)}{R} N_{i}^{\prime}(\phi, \omega) P_{j}(\phi, \omega) \mathrm{d} \phi, \\
& S_{i j}^{r}=\int_{\phi_{0}}^{\phi_{f}} \frac{k A G(\phi)}{R} Q_{i}(\phi, \omega) P_{j}(\phi, \omega) \mathrm{d} \phi \\
& S_{i j}^{s}=\int_{\phi_{0}}^{\phi_{f}} \frac{k A G(\phi)}{R} P_{i}(\phi, \omega) N_{j}^{\prime}(\phi, \omega) \mathrm{d} \phi \\
& S_{i j}^{t}=\int_{\phi_{0}}^{\phi_{f}} \frac{k A G(\phi)}{R} N_{i}^{\prime}(\phi, \omega) N_{j}^{\prime}(\phi, \omega) \mathrm{d} \phi \\
& S_{i j}^{u}=\int_{\phi_{0}}^{\phi_{f}} \frac{k A G(\phi)}{R} Q_{i}(\phi, \omega) N_{j}^{\prime}(\phi, \omega) \mathrm{d} \phi, \\
& S_{i j}^{v}=\int_{\phi_{0}}^{\phi_{s}} \frac{k A G(\phi)}{R} P_{i}(\phi, \omega) Q_{j}(\phi, \omega) \mathrm{d} \phi,
\end{aligned}
$$




$$
\begin{aligned}
S_{i j}^{w} & =\int_{\phi_{0}}^{\phi_{f}} \frac{k A G(\phi)}{R} N_{i}^{\prime}(\phi, \omega) Q_{j}(\phi, \omega) \mathrm{d} \phi, \\
S_{i j}^{x} & =\int_{\phi_{0}}^{\phi_{f}} \frac{k A G(\phi)}{R} Q_{i}(\phi, \omega) Q_{j}(\phi, \omega) \mathrm{d} \phi .
\end{aligned}
$$

The energy dissipated is similarly obtained as

$$
\mathscr{C}=\int_{0}^{T} \sum_{i=1}^{6} \sum_{j=1}^{6} \dot{z}_{i}(t) \dot{z}_{j}(t) C_{i j}(\omega) \mathrm{d} t
$$

where

$$
\begin{aligned}
& C_{i j}=C_{i j}^{a}+C_{i j}^{b}+C_{i j}^{c}, \\
& C_{i j}^{a}=c_{1}(\phi) N_{i}(\phi, \omega) N_{j}(\phi, \omega), \\
& C_{i j}^{b}=c_{2}(\phi) P_{i}(\phi, \omega) P_{j}(\phi, \omega), \\
& C_{i j}^{c}=c_{3}(\phi) Q_{i}(\phi, \omega) Q_{j}(\phi, \omega) .
\end{aligned}
$$

The primes $\left({ }^{\prime}\right)$ in the above equations represent derivatives with respect to the spatial co-ordinate $\phi$. The governing equations for the generalized co-ordinates $z_{k}(t)$ can now be obtained using Lagrange's equations, the $(\cdot)$ denoting the derivative with respect to time:

$$
\frac{\mathrm{d}}{\mathrm{d} t}\left[\frac{\partial \mathscr{L}}{\partial \dot{z}_{k}}\right]-\frac{\partial \mathscr{L}}{\partial z_{k}}=Q_{1_{k}}^{\prime}+Q_{2_{k}}, \quad k=1, \ldots, 6 .
$$

Here, $-Q_{1_{k}}^{\prime}$ are the damping forces, $Q_{2_{k}}$ are the generalized forces, and the Lagrangian is given by $\mathscr{L}(t)=\mathscr{T}(t)-\mathscr{U}(t)$. The stochastic dynamic stiffness matrix is formulated from Lagrange's equation and is thus a function of random variables characterized by the set of dynamic weighted integrals. This leads to the formal representation of discretized equations of motion of the form

$$
\mathbf{M}(\omega) \ddot{\mathbf{z}}(t)+\mathbf{C}(\omega) \dot{\mathbf{z}}(t)+\mathbf{K}(\omega) \mathbf{z}(t)=\overline{\mathbf{f}}(t) .
$$

Here, $\mathbf{M}, \mathbf{C}$ and $\mathbf{K}$ are, respectively, the generalized, frequency-dependent, complex valued, stochastic mass, damping and stiffness matrices. It must be emphasized that these are significantly different from the stiffness, consistent mass and damping matrices encountered in traditional finite element method. Since the definition of dynamic stiffness matrix is essentially with reference to harmonic nodal actions, the forcing function $\overline{\mathbf{f}}(t)$ in the above equation is taken to be of the form $\overline{\mathbf{f}}(t)=\mathbf{F}(\omega) \exp [i \omega t]$. Furthermore, given the fact that the system is linear, it follows that the response vector $\mathbf{z}(t)$ would have the form $\mathbf{z}(t)=\mathbf{Z}(\omega) \exp [i \omega t]$. It can be shown that the forcing vector $\mathbf{F}(\omega)$ and the response vector $\mathbf{Z}(\omega)$ are related to each other through the relation

$$
\mathbf{D}(\omega) \mathbf{Z}(\omega)=\mathbf{F}(\omega)
$$


Here, $\mathbf{D}(\omega)$ is the $6 \times 6$ element dynamic stiffness matrix with elements given by

$$
D_{i j}(\omega)=\left[-\omega^{2} M_{i j}(\omega)+\mathrm{i} \omega C_{i j}(\omega)+K_{i j}(\omega)\right]
$$

while $\mathbf{Z}(\omega)$ and $\mathbf{F}(\omega)$ are, respectively, the vectors of the Fourier transforms of the unknown displacements and applied random forces. Even though the quantities $\mathbf{Z}(\omega)$ and $\mathbf{F}(\omega)$ are, respectively, termed as the Fourier transforms of the functions $\mathbf{z}(t)$ and $\widetilde{\mathbf{f}}(t)$, it must be noted that, strictly speaking, Fourier transforms of samples of stationary random processes do not exist. However, if the functions are defined as $\mathbf{z}(t)=\mathbf{z}(t), \overline{\mathbf{f}}(t)=\overline{\mathbf{f}}(t)$ for $0<t<T_{0}$ and $\mathbf{z}(t)=0, \overline{\mathbf{f}}(t)=0$, for $t>T_{0}$, where $T_{0}$ is a specified value of time $t$, then, as $T_{0} \rightarrow \infty$, the PSD functions of these random processes are well defined, although the sample Fourier transforms do not exist [21].

Since the matrices $\mathbf{M}(\omega), \mathbf{C}(\omega)$ and $\mathbf{K}(\omega)$ are symmetric, it follows that the dynamic stiffness matrix $\mathbf{D}(\omega)$ is also symmetric. The stochastic dynamic stiffness matrix $\mathbf{D}(\omega)$ has a deterministic and a random component and can be represented as

$$
\begin{gathered}
\mathbf{D}(\omega)=\mathbf{D}_{0}(\omega)+\mathbf{D}_{r}(\omega), \\
D_{0_{k l}}(\omega)=-\omega^{2} M_{0_{k l}}(\omega)+\mathrm{i} \omega C_{0_{k l}}(\omega)+K_{0_{k l}}(\omega), \\
D_{r_{k l}}=\sum_{k=1}^{6} \sum_{l=1}^{6} \mathscr{W}_{k l} .
\end{gathered}
$$

Here, the subscripts 0 and $r$, respectively, denote the deterministic and the random parts and $\mathscr{W}_{k l}$ are the weighted integrals. Since $\mathscr{W}_{k l}=\mathscr{W}_{l k}$, the summation in equation (65) occurs only over 21 independent terms $\left(\mathscr{X}_{n}\right)$. Accordingly, equation (65) is re-written in the form

$$
\begin{array}{cc}
D_{k l}=D_{0_{k l}}+\sum_{j=1}^{21} \varphi_{j_{k l}} \mathscr{X}_{j} & (k, l=1, \ldots, 6), \\
\varphi_{n_{k l}}=\Gamma_{k p} \Gamma_{l q} & \text { for } p=q \\
\varphi_{n_{k l}}=\Gamma_{k p} \Gamma_{l q}+\Gamma_{k q} \Gamma_{l p} & \text { for } p \neq q
\end{array}
$$

$\Gamma$ being defined in Appendix A (see equation (A.24)) and

$$
\begin{aligned}
& \mathscr{X}_{1}=\mathscr{W}_{11}, \quad \mathscr{X}_{2}=\mathscr{W}_{12}, \quad \mathscr{X}_{3}=\mathscr{W}_{13}, \quad \mathscr{X}_{4}=\mathscr{W}_{14}, \quad \mathscr{X}_{5}=\mathscr{W}_{15}, \\
& \mathscr{X}_{6}=\mathscr{W}_{16}, \quad \mathscr{X}_{7}=\mathscr{W}_{22}, \quad \mathscr{X}_{8}=\mathscr{W}_{23}, \quad \mathscr{X}_{9}=\mathscr{W}_{24}, \quad \mathscr{X}_{10}=\mathscr{W}_{25}, \\
& \mathscr{X}_{11}=\mathscr{W}_{26}, \quad \mathscr{X}_{12}=\mathscr{W}_{33}, \quad \mathscr{X}_{13}=\mathscr{W}_{34}, \quad \mathscr{X}_{14}=\mathscr{W}_{35}, \quad \mathscr{X}_{15}=\mathscr{W}_{36}, \\
& \mathscr{X}_{16}=\mathscr{W}_{44}, \quad \mathscr{X}_{17}=\mathscr{W}_{45}, \quad \mathscr{X}_{18}=\mathscr{W}_{46}, \quad \mathscr{X}_{19}=\mathscr{W}_{55}, \quad \mathscr{X}_{20}=\mathscr{W}_{56}, \quad \mathscr{X}_{21}=\mathscr{W}_{66} .
\end{aligned}
$$

It is seen that the dynamic stiffness matrix of the beam element is a function of the weighted integrals $\mathscr{X}_{k}(k=1, \ldots, 21)$. The stochastic inhomogeneity in this approximation is thus completely characterized by a set of 21 random variables.

However, if OLE is used for discretizing the random fields, equation (29) is substituted for $f_{k}(\phi)(k=1, \ldots, 8)$ in equation (28). The elements of the stochastic dynamic stiffness matrix 
are now typically of the form

$$
\mathscr{W}_{k l}=\sum_{j=1}^{n} f_{j}\left(\phi_{j}\right) \int_{\phi_{f}}^{\phi_{f}} S_{j}(\phi) N_{k}(\phi, \omega) N_{l}(\phi, \omega) \mathrm{d} \phi .
$$

It must be noted that the integrals appearing in the above equation are deterministic in nature. While this scheme of discretization introduces into the formulation possibly a larger number of random variables as compared to the weighted integral approach, its advantage lies in that the random variables resulting from the discretization of the random fields retain the non-Gaussian probability distributions of the original random fields. This, in turn, is of significance in reliability computations.

\section{RESPONSE ANALYSIS}

For response variability analysis, beam systems driven by stationary excitations are considered. These systems, thus, have two sources of uncertainties; the first is due to the stochastic spatial inhomogeneities of the beam properties, the second is due to the random nature of the external loads. To illustrate the capabilities of the dynamic stiffness matrix approach outlined in the previous section, attention is focussed on the variability in the response PSD due to the uncertainties in structural properties. As a first step, the structure property random fields are discretized and the structural uncertainties are manifested in terms of a vector of random variables. The PSD of the response of the structure, conditioned on these random variables, are evaluated using standard frequency-domain random vibration analysis. The mean and the standard deviation of the response PSD, conditioned on these random variables, are estimated. The response variability analysis is carried out using the three different methods that are to be explained in the following section. It must be noted that the advantage of studying the variability of the response PSD is that it permits a detailed examination of the variability as a function of frequency. Studies on global measures, such as response variance, do not permit such a detailed examination.

\subsection{METHOD 1: NEUMANN'S EXPANSION IN TERMS OF WEIGHTED INTEGRALS}

In this method, the response PSD is computed by inverting the stochastic dynamic stiffness matrix using Neumann's expansion [25]. Assuming the excitation to be a stationary random process, the unknown displacement vector $\mathbf{Z}(\omega)$ is given by

$$
\mathbf{Z}(\omega)=\left[\mathbf{I}-\mathscr{2}(\omega)+\mathscr{Q}^{2}(\omega)-\cdots\right] \mathbf{D}_{0}^{-1}(\omega) \mathbf{F}(\omega),
$$

where $\mathscr{2}(\omega)=\mathbf{D}_{0}^{-1}(\omega) \mathbf{D}_{r}(\omega)$ and $\mathbf{I}$ is the identity matrix. This leads to the PSD matrix for displacements, given by

$$
\begin{aligned}
\mathbf{S}_{Z Z}\left(\omega \mid \mathbf{X}_{0}\right)= & {\left[\mathbf{I}-\mathscr{2}(\omega)+\mathscr{Q}^{2}(\omega)-\cdots\right] \mathbf{D}_{0}^{-1}(\omega) \mathbf{S}_{F F}(\omega) \mathbf{D}_{0}^{-1^{* t}}(\omega) } \\
& {\left[\mathbf{I}-\mathscr{2}(\omega)+\mathscr{Q}^{2}(\omega)-\cdots\right] \mathbf{D}_{0}^{-1^{* 1}}(\omega), }
\end{aligned}
$$

where the operator $(*)$ denotes complex conjugation and the superscript $t$ denotes matrix transpose. The PSD, obtained in equation (71), is conditioned on the system property random variables $\mathbf{X}_{0}$ and hence is itself a random quantity. The variability of the response PSD is estimated by taking expectations across the ensemble of samples and calculating its 
moments. Considering only one-term approximation in the series in equation (71), the first two moments, mean $(\mu)$ and variance $\left(\sigma^{2}\right)$ are, respectively, given by

$$
\begin{aligned}
\mu_{l j}=\left\langle S_{Z Z_{l j}}\left(\omega \mid \mathbf{X}_{0}\right)\right\rangle & =\sum_{m=1}^{6} \sum_{k=1}^{6} \sum_{p=1}^{6} \sum_{q=1}^{6}\left\langle H_{l m} H_{j p}^{*}\right\rangle D_{0_{m k}}^{-1}(\omega) D_{0_{p q}}^{-1^{*}}(\omega) S_{F F_{k q}}(\omega) \\
\sigma_{l j}^{2}= & \sum_{m}^{6} \sum_{k}^{6} \sum_{p}^{6} \sum_{q}^{6} \sum_{a}^{6} \sum_{b}^{6} \sum_{c}^{6} \sum_{d}^{6}\left\langle H_{l m} H_{j p}^{*} H_{i a} H_{j c}^{*}\right\rangle \\
& \times D_{0_{m k}}^{-1}(\omega) D_{0_{p q}}^{-1^{*}}(\omega) D_{0_{a b}}^{-1}(\omega) D_{0_{c d}}^{-1^{*}}(\omega) S_{F F_{k q}}(\omega) S_{F F_{b d}}(\omega)-\mu^{2}
\end{aligned}
$$

where $\left\langle H_{l m} H_{l p}^{*}\right\rangle$ and $\left\langle H_{l m} H_{j p}^{*} H_{l a} H_{j c}^{*}\right\rangle$ are given, respectively, by

$$
\begin{aligned}
\left\langle H_{l m}\right. & \left.H_{l p}^{*}\right\rangle=\delta_{l m} \delta_{l p}+\left\langle\mathscr{Q}_{l m}(\omega) \mathscr{Q}_{l p}(\omega)\right\rangle \\
\left\langle H_{l m} H_{j p}^{*} H_{l a} H_{j c}^{*}\right\rangle= & \delta_{l m} \delta_{l a} \delta_{j p} \delta_{j c}+\delta_{l a} \delta_{j c}\left\langle\mathscr{Q}_{l m} \mathscr{Q}_{j p}\right\rangle+\delta_{l a} \delta_{l m}\left\langle\mathscr{Q}_{j p} \mathscr{Q}_{j c}\right\rangle \\
& +\delta_{l a} \delta_{j p}\left\langle\mathscr{Q}_{j c} \mathscr{Q}_{l m}\right\rangle+\delta_{l a}\left\langle\mathscr{Q}_{j c} \mathscr{Q}_{l m} \mathscr{Q}_{j p}\right\rangle+\delta_{l m} \delta_{j c}\left\langle\mathscr{Q}_{j p} \mathscr{Q}_{l a}\right\rangle \\
& +\delta_{j p} \delta_{j c}\left\langle\mathscr{Q}_{l m} \mathscr{Q}_{l a}\right\rangle+\delta_{j c}\left\langle\mathscr{Q}_{l m} \mathscr{Q}_{j p} \mathscr{Q}_{l a}\right\rangle+\delta_{l m} \delta_{j p}\left\langle\mathscr{Q}_{l a} \mathscr{Q}_{j c}\right\rangle \\
& +\delta_{l m}\left\langle\mathscr{Q}_{j p} \mathscr{Q}_{l a} \mathscr{Q}_{j c}\right\rangle+\delta_{j p}\left\langle\mathscr{Q}_{l m} \mathscr{Q}_{l a} \mathscr{Q}_{j c}\right\rangle \\
& +\left\langle\mathscr{Q}_{l m} \mathscr{Q}_{j p} \mathscr{Q}_{l a} \mathscr{Q}_{j c}\right\rangle .
\end{aligned}
$$

The second, third and fourth order moments of the elements of $\mathscr{Q}$ can further be expressed, respectively, in terms of the second, third and fourth order moments of the weighted integrals $\mathscr{W}$. Thus, it follows that, the evaluation of mean of $S_{Z Z}\left(\omega \mid \mathbf{X}_{0}\right)$ requires knowledge of mean and covariance of $f_{k}(\phi)(k=1, \ldots, 8)$, while the evaluation of the variance of $S_{Z Z}\left(\omega \mid \mathbf{X}_{0}\right)$ demands knowledge of upto fourth order moments. If more than one term is retained in Neumann's expansion, the evaluation of the first two moments of $S_{Z Z}\left(\omega \mid \mathbf{X}_{0}\right)$ would require still higher order moments of $f_{k}(\phi)$. Since the information on these higher order moments are expected to be unavailable, and also for the sake of mathematical expediency, a Gaussian closure assumption is invoked. This allows for the higher order moments of the weighted integrals to be evaluated in terms of mean and covariance of $f_{k}(\phi)$. It is to be noted that this method has three sources of errors arising from: (a) discretization of the random fields, (b) truncation of the Neumann's expansion and (c) Gaussian closure approximation. The last of these errors would not be present if information on higher order moments of $f_{k}(\phi)$ is available.

\subsection{METHOD 2: REDUCED MONTE CARLO SIMULATIONS USING OLE}

In this method, the random fields $f_{k}(\phi)(k=1, \ldots, 8)$ are discretized using OLE. Samples of the random variables obtained by discretizing the random fields are simulated digitally and this leads to sample realizations of dynamic stiffness matrices. These matrices are numerically inverted and an ensemble of response PSD, conditioned on the random variables, is computed. Statistical processing on this ensemble leads to estimates of the mean and variance of $S_{Z Z}\left(\omega \mid \mathbf{X}_{0}\right)$. The sources of errors in this method are those resulting 
from discretization of random fields and the use of limited samples in estimates of mean and variance of $S_{Z Z}\left(\omega \mid \mathbf{X}_{0}\right)$.

\subsection{METHOD 3: FULL-SCALE MONTE CARLO SIMULATIONS}

As has been noted, the methods described in the preceding two sections are approximate in nature. These procedures can be validated by using results from detailed Monte Carlo simulations. This requires the development of a numerical algorithm that generates the sample solutions for the boundary value problem given in equations (20-24). A commonly used strategy in numerical solution of linear boundary problems consists of converting the boundary value problems into a larger class of equivalent initial value problems which, in turn, are amenable for solutions using marching techniques such as Runge-Kutta procedures [26]. Earlier, Manohar and Adhikari [9] have implemented this strategy in their study on dynamic response variability of stochastic Euler-Bernoulli beams using Monte Carlo simulations. In the present study, we follow a similar procedure for analyzing equations (20-24). The details of this formulation are available in the thesis by Gupta [20] and are not provided here. The sources of error in this approach, apart from those associated with the Runge-Kutta integration scheme, are solely associated with the use of limited number of samples in the estimation of response statistics. Moreover, the response calculation procedure used here is independent of the procedure used in methods 2 and 3 . Thus, the results from this method can serve as an acceptable benchmark against which other approximations can be compared.

\section{BUILT-UP STRUCTURES}

The methods developed so far are now extended for computing the response variability of built-up structures. The additional steps needed to characterize the dynamic response of built-up structures are: (1) conversion of the element dynamic stiffness matrix in local co-ordinates into global co-ordinates, (2) assembling of element stiffness matrices in global co-ordinates to form the structure dynamic stiffness matrix, (3) inversion of the random structure dynamic stiffness matrix leading to frequency-domain representation of response, and (4) processing of the Fourier transform of the response variables to arrive at spectral representations of the displacement responses, such as PSD representations. Steps (1) and (2) essentially follow the same rules that are used in the traditional matrix methods of structural analysis. Figures 7 and 8, respectively, show the co-ordinate systems adopted for describing curved and straight beams. The superscripts $g$ and $l$ in these figures denote, respectively, the global and local directions. The element dynamic stiffness matrix in global co-ordinates $\mathbf{D}^{g}(\omega)$ is related to the local dynamic stiffness matrix by the well-known relation

$$
\mathbf{D}^{g}(\omega)=\mathbf{T}^{\mathrm{t}} \mathbf{D}^{l}(\omega) \mathbf{T}
$$

where $\mathbf{T}$ is the transformation matrix. The element dynamic stiffness matrix, in terms of deterministic and random components, is written as

$$
\mathbf{D}^{g}(\omega)=\mathbf{D}_{0}^{g}(\omega)+\mathbf{D}_{r}^{g}(\omega) .
$$

Here, $\mathbf{D}_{0}^{g}(\omega)=\mathbf{T}^{\mathrm{t}} \mathbf{D}_{0}^{l} \mathbf{T}$ is the deterministic part of the element stiffness matrix in the global co-ordinates and $\mathbf{D}_{r}^{g}(\omega)$ is the corresponding random part. In the weighted integral 

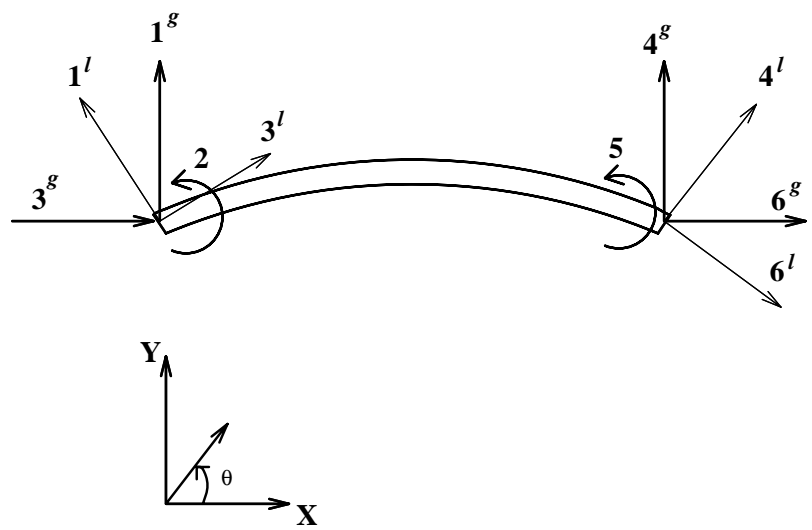

Figure 7. Local and global co-ordinates of the curved beam element: superscript $l$, local axes; $g$, global axes.

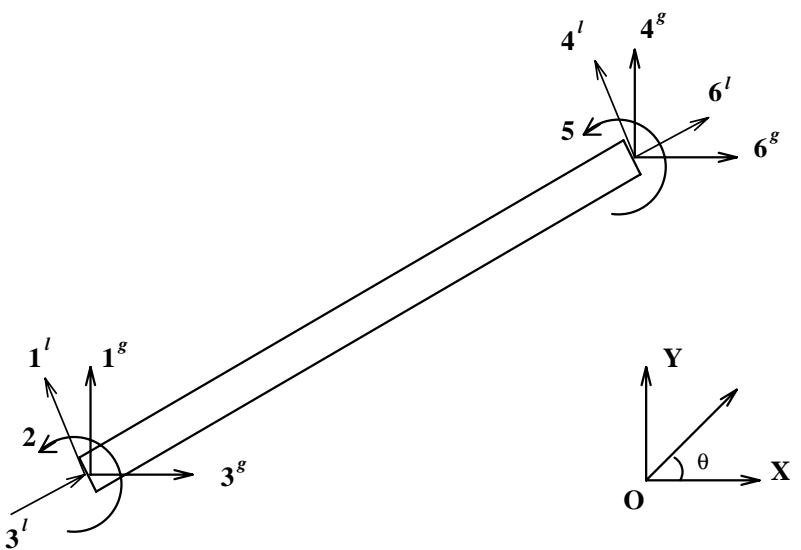

Figure 8. Local and global co-ordinates of the straight beam element: superscript $l$, local axes, $g$, global axes.

approach, equation (66) is written as

$$
D_{k l}^{g}=D_{\mathrm{O}_{k l}}^{g}+\sum_{j=1}^{21} U_{k l}^{j} \mathscr{X}_{j} \quad(k, l=1, \ldots, 6),
$$

where

$$
U_{k l}^{j}=\sum_{p=1}^{6} \sum_{q=1}^{6} \mathbf{T}_{p k} \mathbf{T}_{q l} \varphi_{k l}^{j}
$$

Here, the variable $\varphi_{k l}^{j}$ has the same meaning as in equation (67). In the reduced Monte Carlo simulation approach using OLE, a similar transformation is made.

Formulating the element stiffness matrices in the global co-ordinate system, the matrices are assembled to obtain the global dynamic stiffness matrix. The rules for assembling the global stiffness matrix are identical to those used in the traditional finite element analysis. 
This leads to the expression

$$
\mathbf{K}^{g}(\omega)=\sum_{j=1}^{m} \mathbf{D}_{j}^{g}(\omega)
$$

where $\mathbf{K}^{g}(\omega)$ is the global dynamic stiffness matrix, $\mathbf{D}^{g}$ is the element stiffness matrix in the global co-ordinate system and $m$ is the total number of finite elements in the system. The summation here implies the addition of the appropriate element stiffness matrices at relevant locations. The global system equation can be partitioned in the form

$$
\left[\begin{array}{ll}
\mathbf{K}_{11}^{g}(\omega) & \mathbf{K}_{12}^{g}(\omega) \\
\mathbf{K}_{21}^{g}(\omega) & \mathbf{K}_{22}^{g}(\omega)
\end{array}\right]\left\{\begin{array}{l}
\mathbf{Z}_{k}(\omega) \\
\mathbf{Z}_{u}(\omega)
\end{array}\right\}=\left\{\begin{array}{l}
\mathbf{F}_{u}(\omega) \\
\mathbf{F}_{k}(\omega)
\end{array}\right\}
$$

where $\mathbf{Z}_{k}(\omega)$ and $\mathbf{Z}_{u}(\omega)$, respectively, denote the known and the unknown amplitudes of the nodal harmonic displacements. Similarly, $\mathbf{F}_{u}(\omega)$ and $\mathbf{F}_{k}(\omega)$, respectively, denote the unknown and the known amplitudes of the nodal harmonic forces. In this study, forcing is assumed to be only through the applied nodal forces. Furthermore, it is assumed that the prescribed forces constitute stochastic stationary Gaussian random processes. Accordingly, the equation for the unknown displacements is obtained as

$$
\mathbf{K}_{22}(\omega) \mathbf{Z}_{u}(\omega)=\mathbf{F}_{k}(\omega)
$$

The reduced global stochastic dynamic stiffness matrix can be further written as

$$
\mathbf{K}_{22}(\omega)=\mathbf{K}_{0_{22}}(\omega)+\mathbf{K}_{r_{22}}(\omega),
$$

where $\mathbf{K}_{0_{22}}(\omega)$ is the deterministic part and $\mathbf{K}_{r_{22}}(\omega)$ the stochastic part of the partitioned matrix. To compute the variability in the response, the partitioned dynamic stiffness matrix $\mathbf{K}_{22}(\omega)$ can be inverted using either Neumann expansion or reduced Monte Carlo simulations described in section 4.

\section{RELIABILITY ANALYSIS}

The procedures developed in the earlier sections are now extended to estimate the failure probabilities of inhomogeneous circular Timoshenko beam structures. Two forms of random excitations are considered: the first consists of broadband point excitation and the second, point harmonic excitation with Gaussian amplitude.

\subsection{GAUSSIAN BROADBAND EXCITATIONS}

For Gaussian broadband excitations, the structural response, conditioned on an $n_{x} \times 1$ vector of random variables $\mathbf{X}_{0}$, resulting from the discretization of structure property random fields, is Gaussian. Consequently, results from extreme value theory of Gaussian random processes can be used to evaluate the conditional failure probability. If attention is focussed on the $k$ th displacement component $z_{k}(t)$, it is clear that the pdf of $z_{k}(t)$, conditioned on $\mathbf{X}_{0}$, is also Gaussian. Considering the maximum value $z_{k_{m}}$ over a period of time $T$, given by

$$
z_{k_{m}}=\max _{0<t<T}\left|z_{k}(t)\right|
$$


it can be shown that probability of $z_{k_{m}}<$ threshold value $\alpha$, conditioned on $\mathbf{X}_{0}$, is

$$
P\left[z_{k_{m}}\langle\alpha| \mathbf{X}_{0}\right]=\exp \left[-\frac{1}{2 \pi} \frac{\sigma_{\dot{z}_{k} \mid \mathbf{X}_{0}}}{\sigma_{z_{k} \mid \mathbf{x}_{0}}} T \exp \left\{-\frac{\alpha^{2}}{2 \sigma_{z_{k} \mid \mathbf{x}_{0}}^{2}}\right\}\right],
$$

where

$$
\begin{aligned}
& \sigma_{\dot{z}_{k} \mid \mathbf{X}_{0}}=\int_{-\infty}^{\infty} \omega^{2} S_{Z_{k} Z_{k}}\left(\omega \mid \mathbf{X}_{0}\right) \mathrm{d} \omega, \\
& \sigma_{z_{k} \mid \mathbf{X}_{0}}=\int_{-\infty}^{\infty} S_{Z_{k} Z_{k}}\left(\omega \mid \mathbf{X}_{0}\right) \mathrm{d} \omega
\end{aligned}
$$

with $S_{Z_{k} Z_{k}}\left(\omega \mid \mathbf{X}_{0}\right)$ being computed using the procedures developed in the earlier sections. It should be noted that, in deriving equation (85), it has been assumed that the level crossings are independent and hence constitute a Poisson process [27]. The probability of failure, conditioned on $\mathbf{X}_{0}$, is thus given by

$$
P_{f \mid \mathbf{X}_{0}}=1-P\left[z_{k_{m}}<\alpha \mid \mathbf{X}_{0}\right] .
$$

Consequently, the unconditional probability of failure is obtained as

$$
P\left[z_{k_{m}}>\alpha\right]=\int_{\mathscr{R}}\left\{1-P\left[z_{k_{m}}<\alpha \mid \xi\right]\right\} p_{\mathbf{x}_{0}}(\xi) \mathrm{d} \xi=\left\langle 1-P\left[z_{k_{m}}<\alpha \mid \mathbf{X}_{0}\right]\right\rangle
$$

where $p_{\mathbf{x}_{\mathbf{0}}}(\xi)$ is the $n_{x}$ th order joint pdf of $\mathbf{X}_{0}, \mathscr{R}$ is the domain of integration spanned by $\mathbf{X}_{0}$ and the expectation operator is across the ensemble of $\mathbf{X}_{0}$. It must be noted that since the system property random fields are modelled as non-Gaussian random fields and as the OLE scheme of discretization adopted in the study retain the non-Gaussian features of the random fields, $\mathbf{X}_{0}$ is non-Gaussian. Explicit evaluation of the above expectation still remains a difficult task. However, acceptable solutions can be obtained by using direct Monte Carlo simulations.

\subsection{RANDOM HARMONIC EXCITATIONS}

Since the structure being considered is linear, the response of such systems to harmonic excitations remains harmonic. Consequently, the reliability analysis becomes quasi-static in nature, with the problem becoming frequency dependent instead of being time dependent. The resulting reliability assessment problem is solved based on Monte Carlo simulations that incorporate variance reduction techniques [28-32]. Here, the forcing function is taken to be of the form $\mathbf{F} \exp [\mathrm{i} \omega t]$, where $\mathbf{F}$ is an $\left(n_{f} \times 1\right)$ vector of Gaussian random variables. The $k$ th displacement component, obtained from equation (61), is given by

$$
Z_{k}(\omega ; \mathbf{X})=\sum_{j=1}^{n_{f}} D_{k j}^{-1}\left(\omega ; \mathbf{X}_{0}\right) F_{j}
$$

where $\mathbf{X}$ is an extended vector of random variables, of dimensions $\left(n_{x}+n_{f}\right) \times 1$, defined by $\mathbf{X}=\left[\mathbf{X}_{0}, \mathbf{F}\right]$. Here, $\mathbf{X}_{0}$ and $\mathbf{F}$ are assumed to be stochastically independent. If $\alpha$ is the prescribed limit on $\left|Z_{k}(\omega ; \mathbf{X})\right|$, a performance function

$$
g(\mathbf{X})=\alpha-\max _{\omega_{\min } \leqslant \omega \leqslant \omega_{\max }}\left(\left|Z_{k}(\omega ; \mathbf{X})\right|\right)
$$


can be defined, so that the probability of failure is obtained in terms of an $\left(n_{x}+n_{f}\right)$-dimensional integral as

$$
P_{f}=P[g(\mathbf{X}) \leqslant 0]=\int_{g(x) \leqslant 0} p_{\mathbf{X}}(x) \mathrm{d} x,
$$

where $p_{\mathbf{X}}(x)$ is the $\left(n_{x}+n_{f}\right)$ th order joint pdf of the extended vector of random variables $\mathbf{X}$. As closed-form solutions to this multidimensional integral is not possible, the evaluation of $P_{f}$ is carried out by importance sampling-based simulations. This involves rewriting equation (92) as

$$
P_{f}=\int_{\mathscr{R}^{\prime}} I[g(x) \leqslant 0] \frac{p_{\mathbf{X}}(x)}{h_{\mathbf{X}}(x)} h_{\mathbf{X}}(x) \mathrm{d} x=\left\langle I[g(\mathbf{X}) \leqslant 0] \frac{p_{\mathbf{X}}(\mathbf{X})}{h_{\mathbf{X}}(\mathbf{X})}\right\rangle_{h_{\mathbf{X}}} .
$$

Here, $I[\cdot]$ is an indicator function that is equal to 1 if $g(\mathbf{X}) \leqslant 0$ and 0 otherwise, $h_{\mathbf{X}}(x)$ is the $\left(n_{x}+n_{f}\right)$ th order importance sampling density function, $\langle\cdot\rangle_{h_{\mathbf{X}}}$ denotes expectation with respect to $h_{\mathbf{X}}(x)$ and $\mathscr{R}^{\prime}$ is the region spanned by the random variables $\mathbf{X}$.

In order to obtain a starting sampling function for a target threshold level $\alpha_{T}, h_{\mathbf{X}}(x)$ is usually assumed to be Gaussian and is centred around the design point obtained by computing the reliability index [30,31]. This study, however, avoids reliability index calculations. Instead, a first guess of the sampling density function is obtained by fitting a probability density function for the samples lying in the failure domain, obtained from a pilot simulation run for a low threshold level $\alpha_{0}$. In the subsequent simulation cycles, as $\alpha$ is varied in increments of $\mathrm{d} \alpha$ towards the target threshold level, the sampling function is modified to reflect the increase in knowledge of the failure domain resulting from each simulation run. The details of the steps adopted in this sampling scheme are outlined here:

1. As a starting point, $\alpha$ is first set to its lowest limit of interest, $\alpha_{0}$. A pilot simulation run is carried out to generate $N_{0}$ samples of the $\left(n_{x}+n_{f}\right)$-dimensional vector random variables $\mathbf{X}$.

2. Let $N_{1}$ of these samples lie in the failure domain. Using these $N_{1}$ samples, the mean and covariance matrices, respectively, of dimensions $\left(n_{x}+n_{f}\right) \times 1$ and $\left(n_{x}+n_{f}\right) \times$ $\left(n_{x}+n_{f}\right)$, conditioned on $g(\mathbf{X}) \leqslant 0$, given by

$$
\begin{gathered}
\{\mu\}=\langle\mathbf{X} \mid g(\mathbf{X}) \leqslant 0\rangle, \\
{\left[\mathbf{C}_{\mathbf{0}}\right]=\left\langle\{\mathbf{X}-\mu\}\{\mathbf{X}-\mu\}^{\mathrm{t}} \mid g(\mathbf{X}) \leqslant 0\right\rangle}
\end{gathered}
$$

are estimated.

3. The mean $\mu_{k}$ and variance $\sigma_{k}^{2}\left(k=1, \ldots, n_{x}\right)$ estimated from equations $(94,95)$, together with the knowledge of the range $\left(a_{k}, b_{k}\right)\left(k=1, \ldots, n_{x}\right)$ of the random variables $X_{1}, \ldots, X_{n_{k}}$, are used to construct the first order pdf's of these random variables, by invoking the principle of maximum entropy. These first order pdf's are of the form as given in equation (12). The first order pdf's of the remaining random variables $X_{n_{x}+1}, \ldots, X_{n_{f}}$ are taken to be Gaussian with mean $\mu_{k}$ and variance $\sigma_{k}^{2}$ $\left(k=n_{x}+1, \ldots, n_{f}\right)$ estimated from equations $(94,95)$. Furthermore, the first order pdf models are combined with the estimated covariance matrix $\left[\mathbf{C}_{0}\right]$ to obtain an $\left(n_{x}+n_{f}\right)$ th order Nataf's model for $h_{\mathbf{x}}(x)$ (see equation (13)). It must be noted that $\mathbf{X}_{0}$ and $\mathbf{F}$ do not remain uncorrelated when conditioned on the event $[g(\mathbf{X}) \leqslant 0]$. 
4. $N_{2}$ number of samples are generated using the sampling density $h_{\mathbf{X}}(x)$. The failure probability $P_{f}$ is estimated as an average, as described in equation (93).

5. Steps 2-5 are repeated till convergence in $P_{f}$, corresponding to a particular threshold level $\alpha_{j}$, is achieved. The number of samples $N_{1}$ in step 2 is now obtained from simulations carried out in step 4.

6. Next, the failure surface is redefined by changing $\alpha_{j}$ to $\alpha_{j+1}=\alpha_{j}+\mathrm{d} \alpha$. Since the variance of the samples lying in the failure domain are small, the samples generated by $h_{\mathbf{x}}(x)$ do not fall in the new failure domain. This difficulty can be overcome by setting the variance of the $n_{f}$ Gaussian random variables $X_{n_{x}+1}, \ldots, X_{n_{f}}$ to arbitrarily higher values [32]. The first order pdf's for these random variables are constructed using these variances but without changing the mean computed from equation (94). A new sampling density function $h_{\mathbf{X}}(x)$ is computed by repeating step 3. Steps $2-5$ are repeated till convergence in $P_{f}$, for the new threshold level $\alpha_{j+1}$, is achieved.

7. Steps $2-6$ are repeated till the failure probability corresponding to the target threshold level $\alpha_{T}$ is estimated.

\section{NUMERICAL EXAMPLES AND DISCUSSIONS}

For illustration of the proposed methods, two structures are considered. The first is a propped cantilever circular Timoshenko beam fixed at one end and hinged at the other end and the second structure is as shown in Figure 13. In both these examples, the quantities $E(\phi), \rho(\phi), G(\phi), b(\phi), d(\phi), c_{1}(\phi), c_{2}(\phi)$ and $c_{3}(\phi)$ are modelled as mutually independent, homogeneous, stochastic fields. The quantities $f_{k}(\phi)(k=1, \ldots, 8)$ are taken to be bounded in the region $a_{k}=-\sqrt{3}$ and $b_{k}=\sqrt{3}$ with $\varepsilon_{k}=0.05$ and $\left\langle f_{k}\right\rangle=0$. This would mean that the mean values of the beam parameters $E(\phi), G(\phi), \rho(\phi), b(\phi), d(\phi), c_{1}(\phi), c_{2}(\phi)$ and $c_{3}(\phi)$, listed in equation (7), are equal to their respective nominal values $E_{0}, G_{0}, \rho_{0}, b_{0}, d_{0}, c_{1_{0}}$, $c_{20}$ and $c_{3_{0}}$. The parameters in the maximum entropy distribution in equation (12) are obtained as $\lambda_{1_{k}}=0.3596, \lambda_{2_{k}}=0.99 \times 10^{-3}$ and $\lambda_{3_{k}}=0.1548(k=1,8)$. Furthermore, the autocovariance for $f_{k}(\phi)(k=1, \ldots, 8)$ are all taken to be of the form $R(\tau)=\exp \left[-\alpha \tau^{2}\right]$. This form of autocovariance satisfies all the requirements stated in equations (9-11). The correlation length associated with this form of autocovariance function can be shown to be given by $\frac{1}{2} \sqrt{\pi / \alpha}$ with $\alpha$ being the correlation parameter. In the numerical work, $\alpha$ is so selected that this correlation length becomes $1 / 4$ th of the spatial extent of the associated beam element. To determine the number of terms, $n$, needed to represent $f_{k}(\phi)$ by OLE, the discretization error was computed as a function of the number of terms. It was observed that the discretization error was 0.075, 0.016 and 0.004, respectively, for 5, 6 and 7 number of terms. Figure 9 shows the covariance of $\tilde{f}(\phi)$ with $n=6$, compared with the target covariance function of $f_{k}(\phi)$. In the numerical work, $n$ is taken to be 6 and it was observed that the global discretization error in this case is 0.016 , which is considered acceptable. Finally, the methods for estimating failure probabilities, described in section 6, are illustrated by considering the propped cantilever circular Timoshenko beam subjected to two alternative classes of excitations: broadband excitations and random harmonic forces.

\subsection{EXAMPLE 1: DETERMINISTIC CURVED TIMOSHENKO BEAM}

A curved Timoshenko beam, fixed at one end and hinged at the other, with a span of $100 \mathrm{~m}$, radius of curvature $R=82.03 \mathrm{~m}$ and mean cross-sectional dimensions of $0.35 \mathrm{~m} \times 0.335 \mathrm{~m}$ 


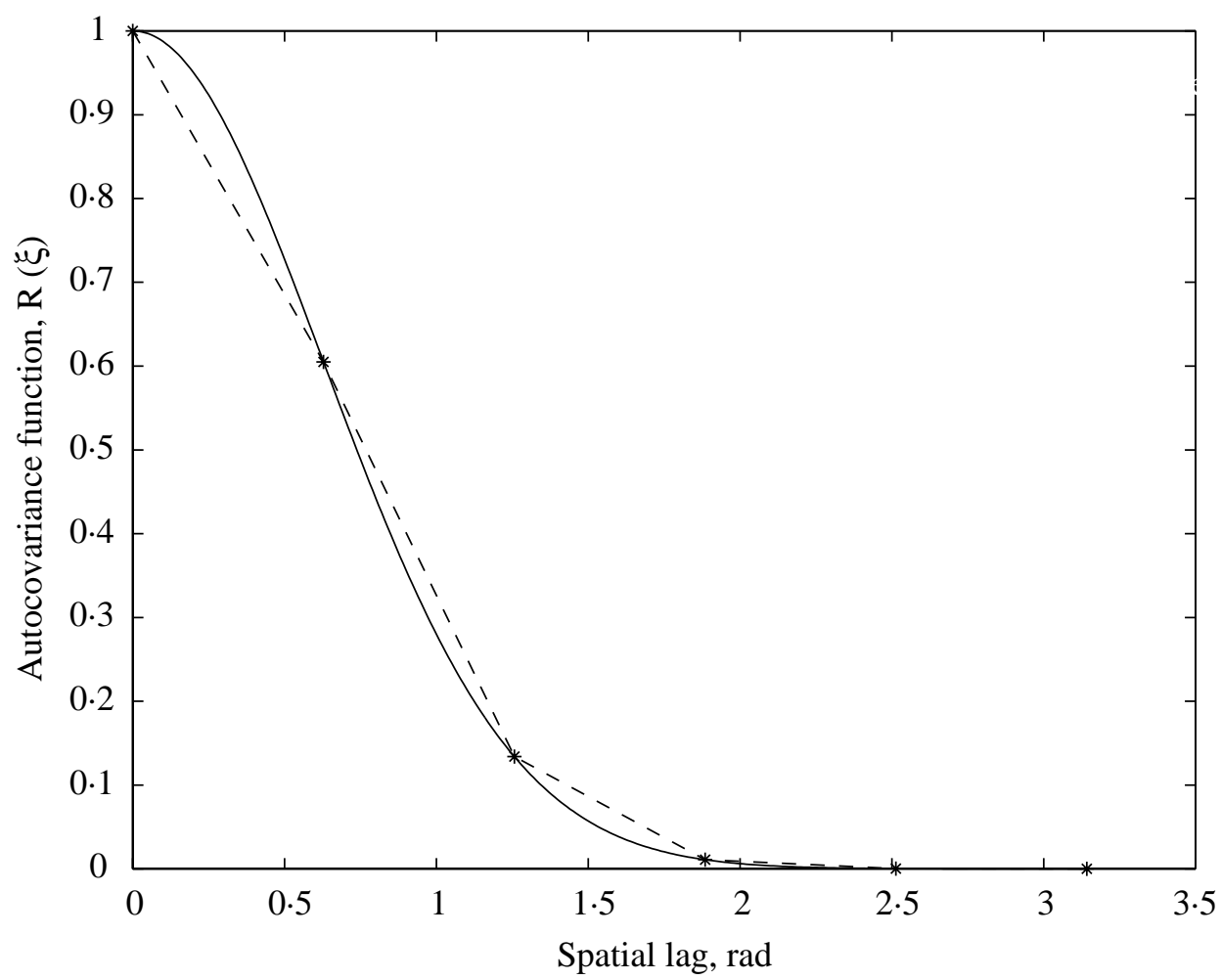

Figure 9. Autocovariance function of $\tilde{f}(\phi)$ using OLE, $\phi_{0}=0 \mathrm{rad}, \phi_{f}=\pi \mathrm{rad}:-$, target; - $-\star-$, discretized.

is considered. Nominal values of the system properties are taken as follows: $E_{0}=2 \cdot 1 \times 10^{11} \mathrm{~N} / \mathrm{m}^{2}, \quad \rho_{0}=2850 \mathrm{~kg} / \mathrm{m}^{3}, \quad G_{0}=8 \cdot 0769^{10} \times \mathrm{N} / \mathrm{m}^{2}$, and $c_{1_{0}}=c_{2_{0}}=c_{3_{0}}=$ $160 \mathrm{Ns} / \mathrm{m}$. A harmonic bending moment $\exp [\mathrm{i} \omega t]$ is applied at the hinged end and the resultant rotation at the hinged end is computed using the following four independent approaches: (1) direct dynamic stiffness matrix method where the dynamic stiffness matrix is obtained in closed form by applying harmonic loads and displacements at the nodes and subsequently eliminating the vector of constants required to satisfy the prescribed boundary conditions [22]; (2) frequency-dependent shape functions to formulate the dynamic stiffness matrix which has been subsequently used to obtain the structural response (section 3.3); (3) a commercial finite element code, such as NISA; and (4) a numerical integration scheme referred to in section 4.3. The 4th order Runge-Kutta numerical integration scheme, with modification due to Gill, was used with a step size $0 \cdot 1 \mathrm{rad}$. The finite element model used comprised of 100 straight beam elements. Normal mode expansions considering 100 modes were used for calculating the response of the structure. The response obtained by the four methods are illustrated in Figure 10. The results are shown to be in reasonable mutual agreement with each other. This provides the confidence that the procedures developed are correct and are implemented correctly. The frequency response function is observed to consist of alternating sequences of resonant peaks and antiresonant dips as might be expected in a direct receptance plot. The first few natural frequencies of the system are observed to be at $3 \cdot 3,6 \cdot 8,12 \cdot 5,18 \cdot 8,27 \cdot 3,36 \cdot 4$ and $47 \cdot 8 \mathrm{rad} / \mathrm{s}$ respectively. It was observed that the convergence of finite element results, especially at antiresonant frequencies, was slow with respect to the number of finite elements used. 


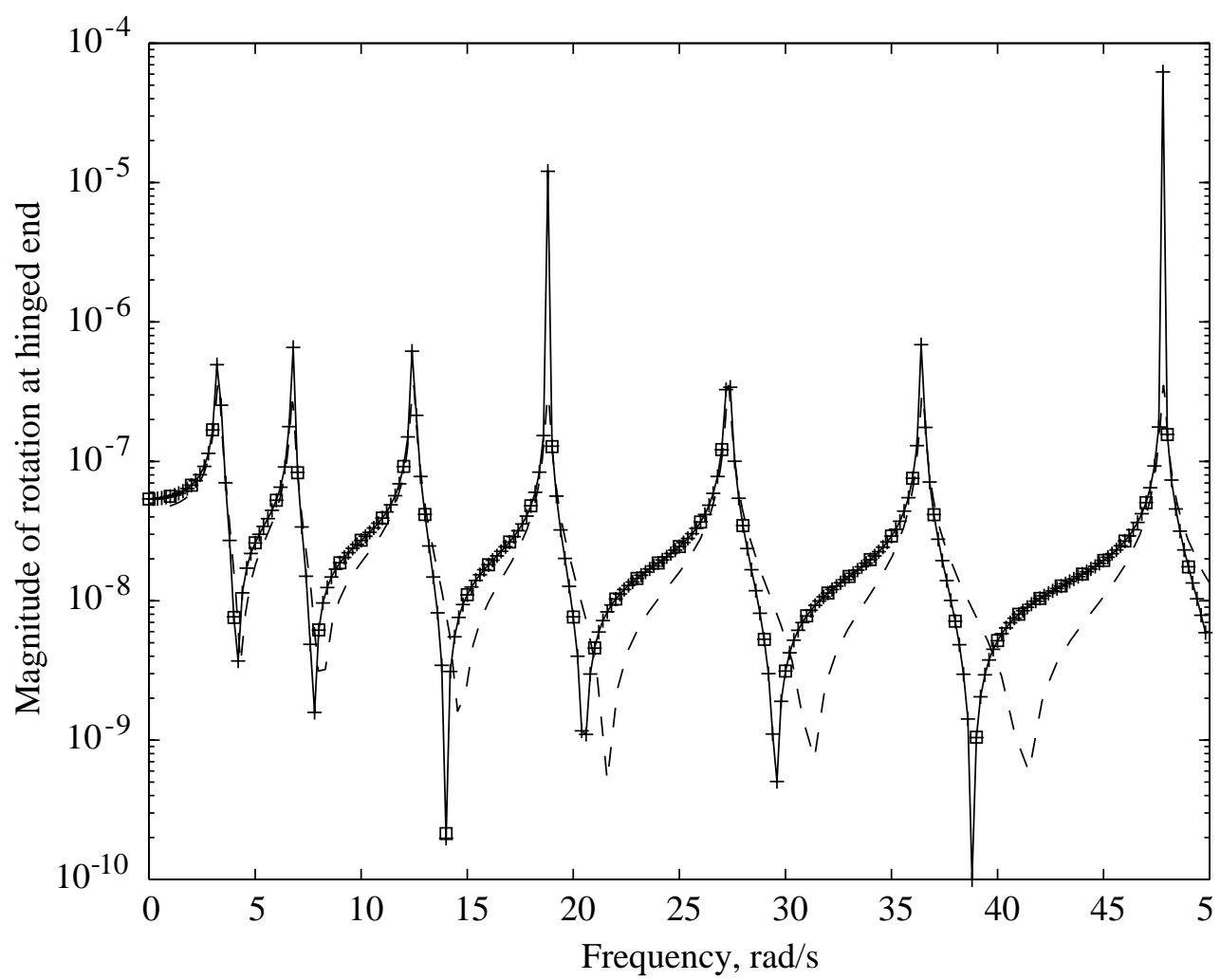

Figure 10. Example 1. Comparison of response at d.o.f. 5 of deterministic curved beam:----, FEM with 100 elements; — , DSM using shape functions; + + , direct DSM approach; $\square \square$, numerical integration.

\subsection{EXAMPLE 2: RANDOMLY INHOMOGENEOUS CURVED TIMOSHENKO BEAM}

The nominal structure considered in example 1 is now perturbed by stochastic inhomogeneities with properties as outlined in the opening of this section. A stationary random bending moment, characterized by an input PSD, is applied at the hinged end and the variability in the PSD of rotation at the hinged end is studied using the three methods discussed in section 4 . It must be noted that the reduced system dynamic stiffness matrix has only one entry, namely $D_{55}(\omega)$. Consequently, issues related to inversion of the dynamic stiffness matrix becomes trivially simple. The mean and the standard deviation of the response PSD are plotted in Figures 11 and 12. The results from alternative methods are observed to be in good agreement with each other. This lends credence to the approximations made in the development of the proposed methods. A comparison with Figures 11 and 12 reveal that the peaks and dips occur at the same frequencies as observed in Figure 10 indicating that there are no appreciable changes in the natural frequencies due to the uncertain fluctuations in the system parameters. It must be noted that an ensemble size of 500 samples was used in the Monte Carlo simulations. Issues related to the inversion of stochastic dynamic stiffness matrix are illustrated in the example considered next.

\subsection{EXAMPLE 3: BUILT-UP STRUCTURE}

A built-up structure (Figure 13) is considered consisting of two unequal straight beams of lengths 10 and $8 \mathrm{~m}$, respectively, and a curved semicircular beam with radius $R=10 \mathrm{~m}$. The 


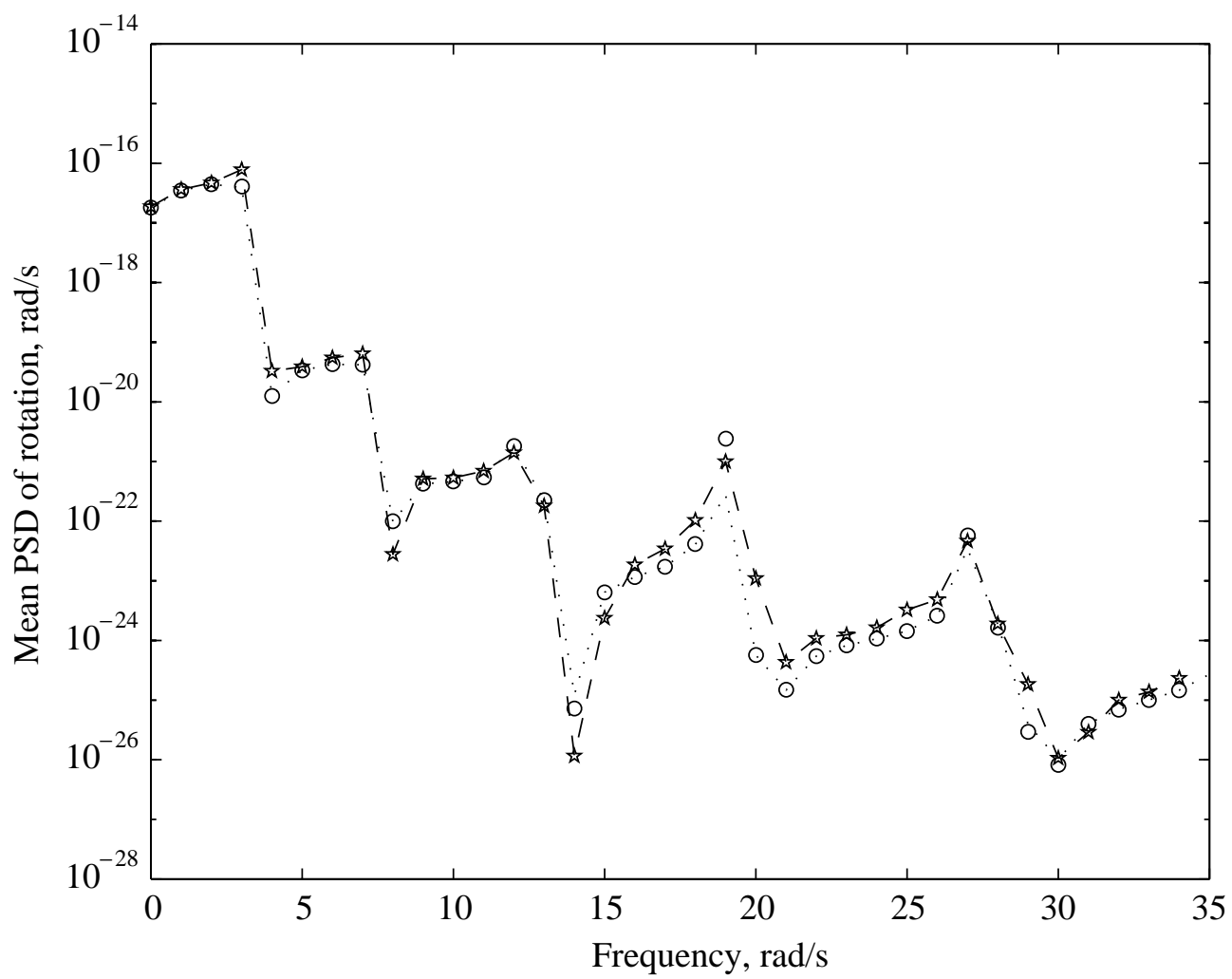

Figure 11. Example 2. Mean of PSD of response at node $5 ;-\star-$, method $1 ; \bigcirc$, method $2 ; \cdots \cdots$, method 3.

assumed nominal values for all the three beam segments are: $b_{0}=0.3 \mathrm{~m}, d_{0}=0.1 \mathrm{~m}$, $E_{0}=2 \cdot 1 \times 10^{11} \mathrm{~N} / \mathrm{m}^{2}, \rho_{0}=2850 \mathrm{~kg} / \mathrm{m}^{3}, G_{0}=8.0769 \times 10^{10} \mathrm{~N} / \mathrm{m}^{2}$ and $c_{1_{0}}=c_{2_{0}}=c_{3_{0}}=$ $160 \mathrm{Ns} / \mathrm{m}$. The stochastic variations are, again, as described in the opening of this section. A steady state excitation force, characterized by an input PSD, is applied along d.o.f. 4. The reduced global dynamic stiffness matrix is of order $6 \times 6$. The variability in the PSD for the response along d.o.f. 4 are estimated using the three methods discussed in section 4 . Figures 14 and 15 show that the results obtained by the three methods are in fairly good agreement with each other. This example also validates the use of Neumann expansion series with the attendant Gaussian closure approximations as means for estimating the moments of inverse of a random matrix. It must be noted that in the numerical work, a single-term approximation was considered in the Neumann expansion.

\subsection{EXAMPLE 4: FIRST PASSAGE FAILURE FOR CURVED TIMOSHENKO BEAM}

The propped cantilever circular Timoshenko beam considered in example 2 is now assumed to be subjected to stationary broadband bending moment at the hinged end. For the purpose of illustration, only mass distribution is considered to have random fluctuations. This limited the number of correlated non-Gaussian random variables entering the formulation to 6 . The first passage failure probability, conditioned on $\mathbf{X}_{0}$, is estimated for the rotation at the hinged end and the unconditional failure probability was 


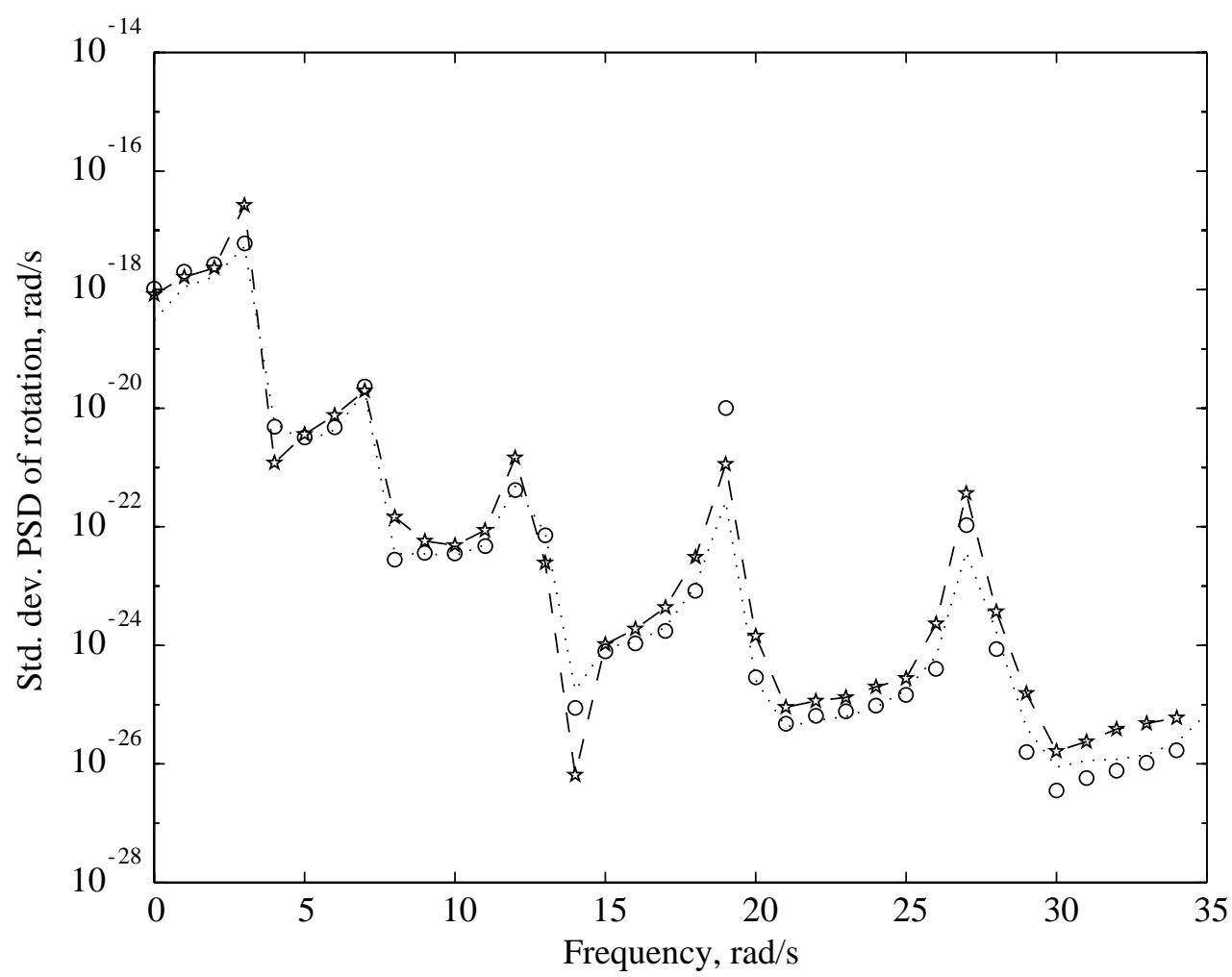

Figure 12. Example 2. Standard deviation of PSD of response at node $5 ;-\star-$, method $1 ; \bigcirc$, method $2 ; \cdots \cdots$, method 3.

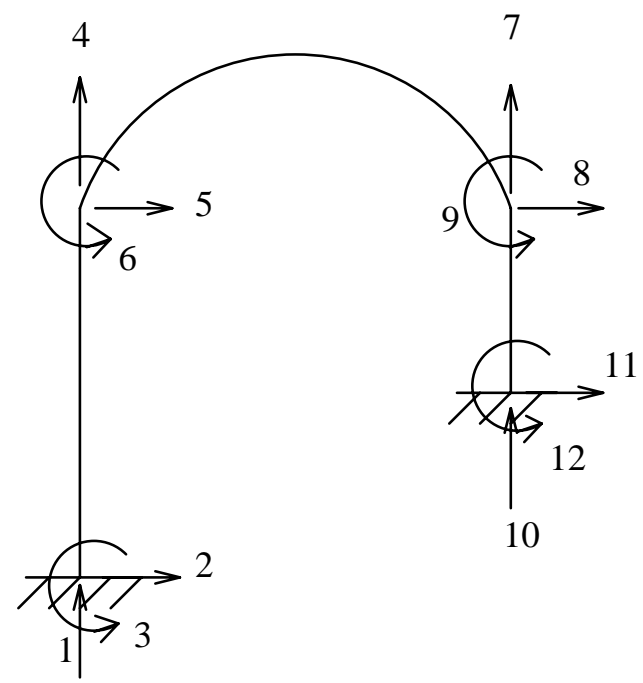

Figure 13. Built-up structure comprising of straight and curved Timoshenko beam elements.

determined by direct Monte Carlo simulations using 1000 samples. The variation of the failure probability as a function of threshold level $\alpha$ is illustrated in Figure 16. The failure probability curve for the corresponding beam, when the random fluctuations in the beam 


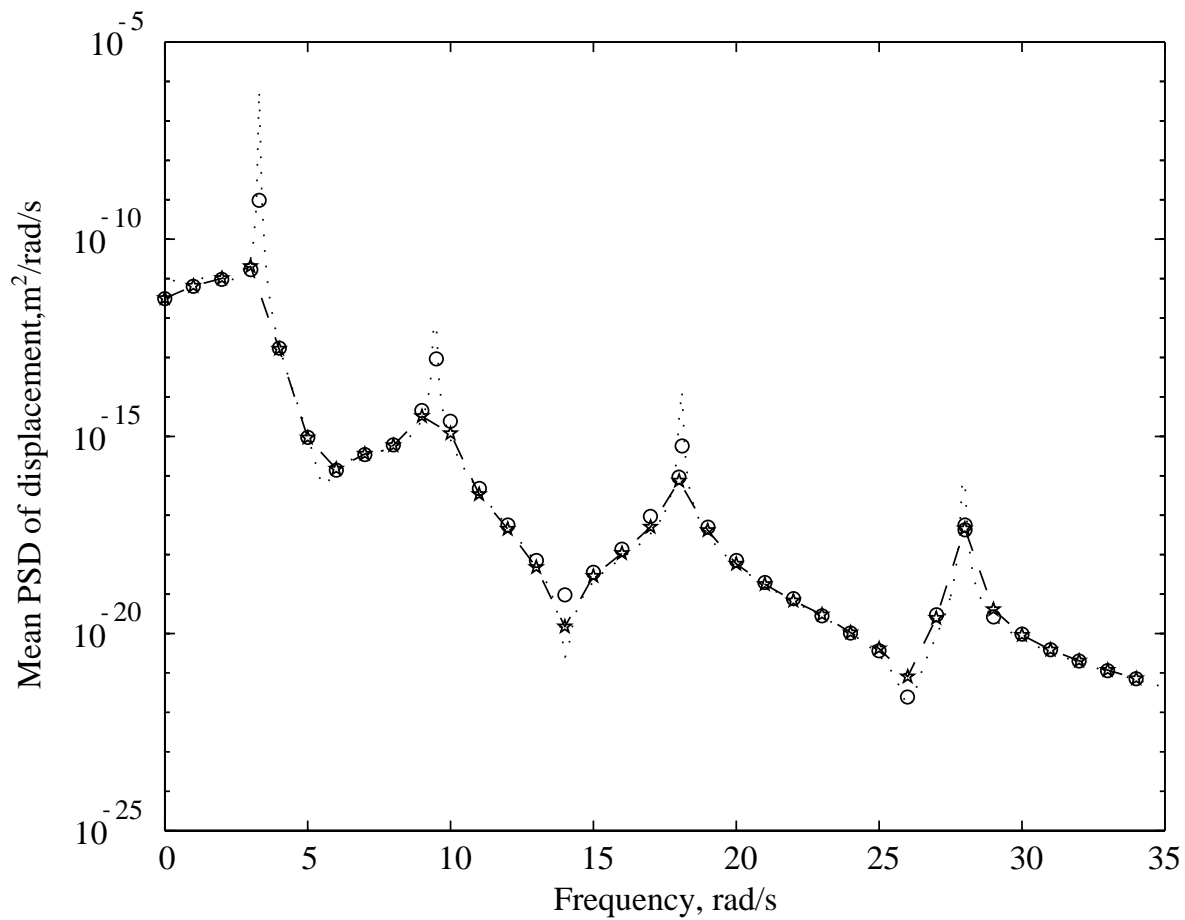

Figure 14. Example 3. Mean of PSD of displacement at node 4; $-\star-$, Method 1; $\bigcirc$, method 2, $\cdots$, method 3.

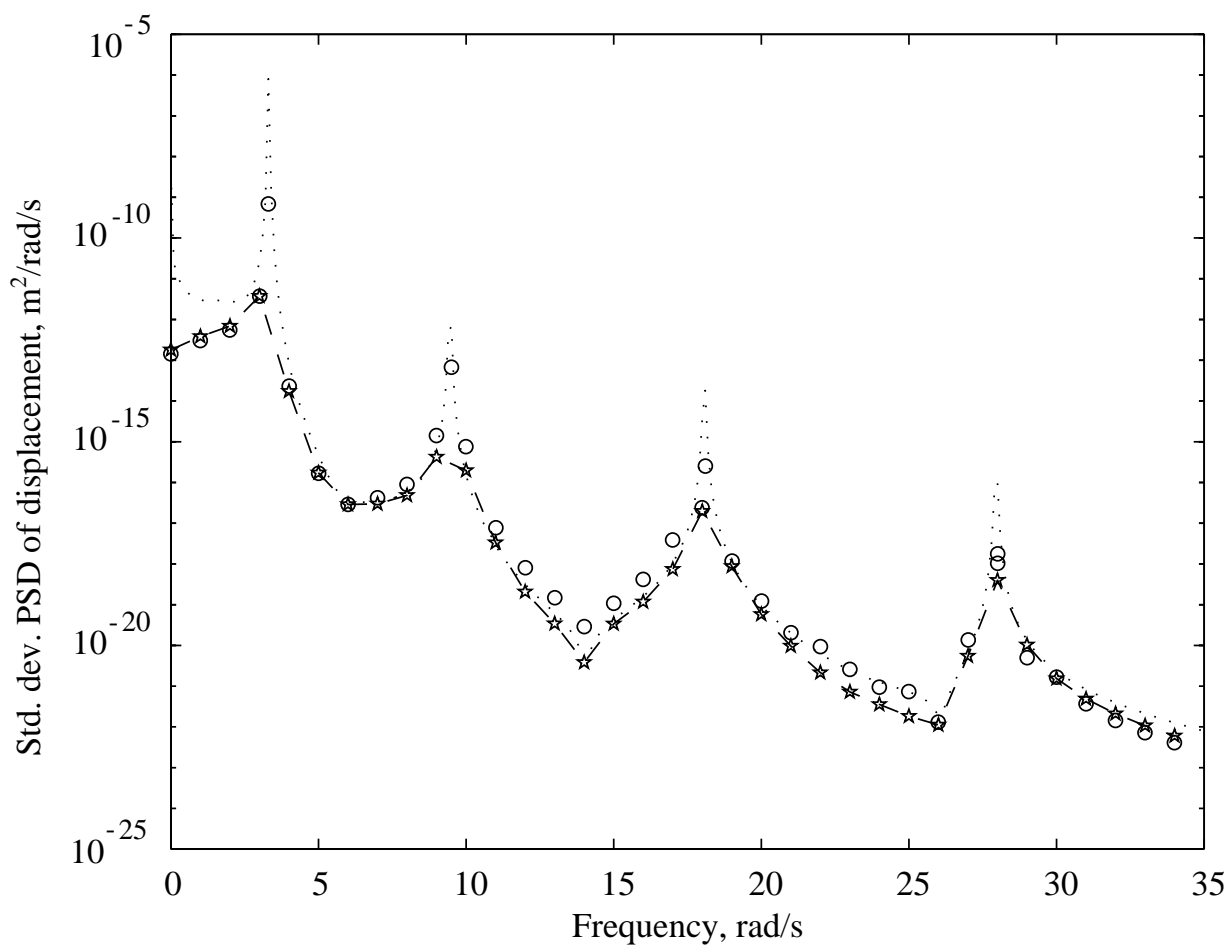

Figure 15. Example 3. Standard deviation of PSD of displacement at node 4: $-\star-$, method 1; $\bigcirc$, method 2; $\cdots$, method 3 . 


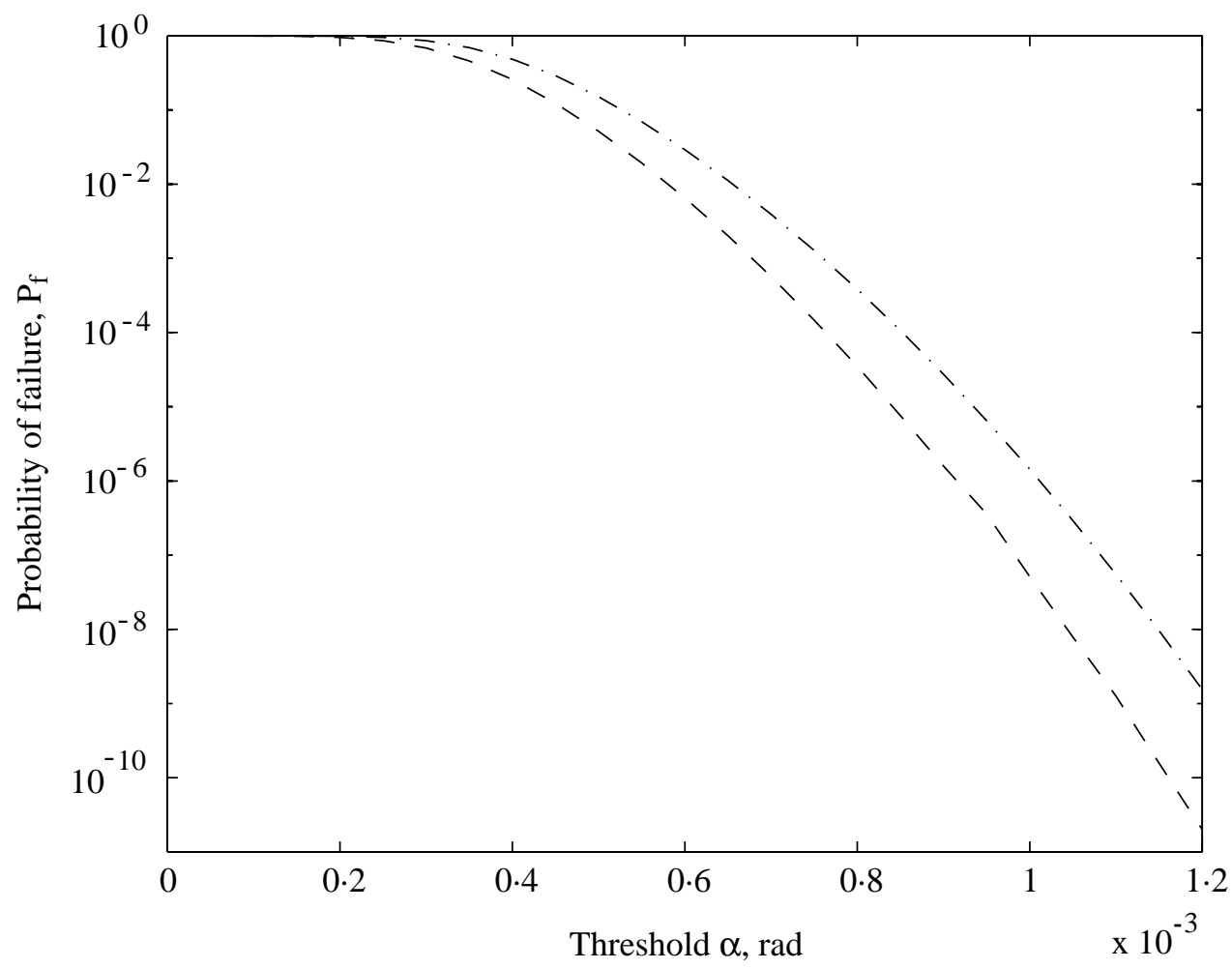

Figure 16. Example 4. First passage failure probability, excitation is broad banded; —- , randomly inhomogeneous beam, $\cdot \cdot \cdot-\cdot-\cdot-$, deterministic beam.

properties are neglected, is also shown in this figure. It is seen that the effect of considering system uncertainties is to reduce the probability of failure.

\subsection{EXAMPLE 5: FAILURE PROBABILITY UNDER RANDOM HARMONIC EXCITATION}

A harmonic bending moment $F \exp [\mathrm{i} \omega t]$, with amplitude $F$ modelled as a Gaussian random variable, is assumed to act at the hinged end in the structure considered in the previous example. As in the previous example, only mass is taken to be stochastic in nature. The excitation moment $F$ is modelled as a Gaussian random variable with mean $10 \mathrm{kN} \mathrm{m}$ and standard deviation $10 \times 0.05 \mathrm{kN} \mathrm{m}$. The performance function is as defined in equation (91) with $\alpha$ taken to range from 0.0017 to $0.011 \mathrm{rad}$. The initial Monte Carlo simulation run was performed for threshold value $\alpha=0.0017 \mathrm{rad}$ with 1000 samples. The estimation of failure probabilities for higher values of $\alpha$ subsequently employed importance sampling, using non-Gaussian sampling functions. The number of samples for each simulation run was taken to be 500. Figure 17 shows the resulting estimates of probability of failure as a function of threshold value $\alpha$. The graph also shows variation of $P_{f}$ with $\alpha$ when beam is taken to be deterministic. The procedure has enabled calculation of $P_{f}$ as low as $10^{-10}$ with the total number of simulation cycles for this level of $P_{f}$ being of the order of only about $10^{4}$. The trend of variation of $P_{f}$ with $\alpha$ for the cases of deterministic beam and beam with random properties is seen to be different from what was observed in the previous example. 


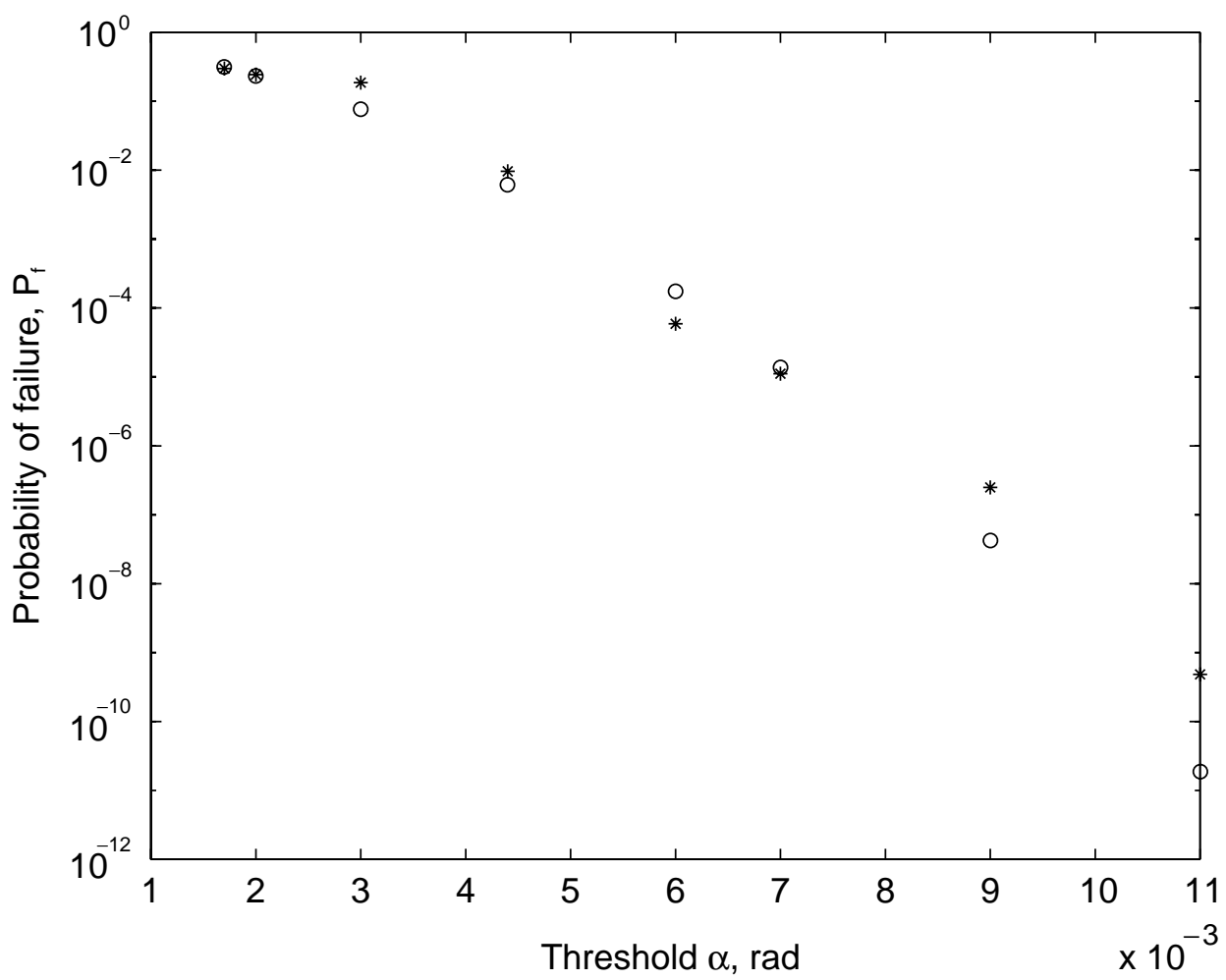

Figure 17. Example 5. Failure probability using adaptive importance sampling, random harmonic excitations; $\star$, randomly inhomogeneous beam; $\bigcirc$, deterministic beam.

For most values of $\alpha$, but not for all $\alpha$, it is observed that the effect of beam randomness is to increase the $P_{f}$.

\section{CONCLUSIONS}

A frequency-domain stochastic finite element approach is outlined for the vibration analysis of skeletal structures made up of randomly inhomogeneous, curved/straight Timoshenko beams. The study extends deterministic dynamic stiffness matrix methods to problems of structural system stochasticity. Issues related to response variability analysis and reliability assessments are addressed. The following are the major conclusions based on this study. (1) The use of frequency-dependent shape functions relieves the dependence of finite element mesh size on frequency range of excitation. Also, the dependence of shape functions on damping permits a satisfactory treatment of damping in dynamic response analysis. (2) The optimal linear expansion scheme offers a powerful means to discretize non-Gaussian random fields, especially in studies involving reliability assessment. This scheme retains at the nodal points the first order probability distribution characteristics and covariance structure of non-Gaussian random fields being discretized. (3) The weighted integral approach for discretizing random fields, in principle, is capable of taking into account the non-Gaussian features of the random fields provided descriptions of higher order moments of these random fields are available. The difficulties associated with obtaining probability density function of the weighted integrals make this method of 
discretization to be of limited relevance in problems of reliability assessment. (4) Both weighted integrals and optimal linear expansion schemes perform equally well, insofar as problems of variability assessment are concerned. (5) For randomly driven systems, the influence of system stochasticity on probability of failure is observed to depend on the nature of excitation. For broadband excitations, the system randomness is observed to lower the probability of failure, while for random harmonic inputs, the probability of failure is seen in most but not all cases, to increase the probability of failure. It is felt that further research is required to clarify this issue.

\section{REFERENCES}

1. G. I. SCHUELLER (editor) 1997 Probabilistic Engineering Mechanics 12, 198-321. A state of the art report on computational stochastic mechanics.

2. C. S. Manohar and R. A. Ibrahim 1999 American Society of Mechanical Engineers Applied Mechanics Reviews 52, 177-197. Progress in structural dynamics with stochastic parameter variations.

3. E. Vanmarcke, M. Shinozuka, S. Nakagiri, G. I. Schueller and M. Grigoriu 1986 Structural Safety 3, 143-166. Random fields and stochastic finite elements.

4. C. G. Bucher and C. E. Brenner 1992 Archive of Applied Mechanics 62, 507-516. Stochastic response of uncertain systems.

5. A. Der Kiureghian and J. B. Ke 1988 Probabilistic Engineering Mechanics 3, 83-91. The stochastic finite element method in structural reliability.

6. C. E. BRENNER 1991 Internal Working Report No. 35-91, Institute of Engineering Mechanics, University of Innsbruck, Austria. Stochastic finite element methods.

7. R. Ghanem and P. D. Spanos 1991 Stochastic Finite Elements: A Spectral Approach. New York: Springer-Verlag.

8. M. Kleiber and T. D. Hein 1992 The Stochastic Finite Element Method. Chichester: John Wiley.

9. C. S. Manohar and S. AdHIKARI 1998 Probabilistic Engineering Mechanics 13, 39-52. Dynamic stiffness matrix of randomly parametered beams.

10. S. ADHIKARI and C. S. MANOHAR 1999 International Journal of Numerical Methods in Engineering 44, 1157-1178. Dynamic analysis of framed structures with statistical uncertainties.

11. S. AdHIKARI and C. S. MANOHAR 2000 American Society of Civil Engineers Journal of Engineering Mechanics 126, 1131-1140. Transient dynamics of stochastically parametered beams.

12. M. SHINOZUKA 1987 American Society of Civil Engineers Journal of Engineering Mechanics 113, 825-842. Structural response variability.

13. C. G. BUCHER and M. SHINOZUKA 1988 American Society of Civil Engineers Journal of Engineering Mechanics 114, 2035-2054. Structural response variability II.

14. T. TAKADA 1990 Probabilistic Engineering Mechanics 5, 146-165. Weighted integral method in stochastic finite element analysis.

15. G. DeOdatis 1991 American Society of Civil Engineers Journal of Engineering Mechanics 117, 1851-1864. Weighted integral method I: stochastic stiffness matrix.

16. C. S. Manohar, S. Adhikari and S. Bhattacharyya 1999 Statistical Energy Analysis of Structural Dynamical Systems, Department of Science and Technology Report, Project Number III 5(1)/95-ET. Monte Carlo simulations of steady and transient dynamics of beams with spatially inhomogeneous non-Gaussian parameter uncertainties.

17. M. GrIGORIU 1984 American Society of Civil Engineers Journal of Engineering Mechanics 110, 610-620. Crossings of non-Gaussian translation processes.

18. A. Der KiUReghian and P. L. LiU 1986 American Society of Civil Engineers Journal of Engineering Mechanics 112, 85-104. Structural reliability under incomplete probability information.

19. C. Li and A. Der KiUReghian 1993 American Society of Civil Engineers Journal of Engineering Mechanics 119, 1136-1153. Optimal discretization of random fields.

20. S. Gupta 2000 M.Sc. Engineering Thesis, Indian Institute of Science, Bangalore, India. http://civil.iisc.ernet.in/ sayan/pub.html. Vibration analysis of structures built-up of randomly inhomogeneous curved and straight beams using stochastic dynamic stiffness matrix method. 
21. A. Papoulis 1997 Probability, Random Variables and Stochastic Processes. New York: McGraw-Hill.

22. M. S. Issa, T. M. WANG and B. T. HsiaO 1987 Journal of Sound and Vibration 114, 297-308. Extensional vibrations of continuous circular curved beams with rotary inertia and shear deformation. Part I: free vibration.

23. O. Ditlevsen, G. Mohr and P. Hoffmeyer 1996 Probabilistic Engineering Mechanics 11, 15-23. Integration of non-Gaussian fields.

24. G. Mohr 1999 Probabilistic Engineering Mechanics 14, 137-140. Higher moments of weighted integrals of non-Gaussian fields.

25. F. Yamazaki, M. Shinozuka and G. Dasgupta 1988 American Society of Civil Engineers Journal of Engineering Mechanics 114, 1335-1354. Neumann expansion for stochastic finite element analysis.

26. F. B. Hilderbrand 1974 Introduction to Numerical Analysis, New Delhi: Tata-McGraw Hill.

27. N. C. NigAM 1983 Introduction to Random Vibrations. Cambridge, Massachussets: MIT Press.

28. H. KAHN 1956 in Symposium on Monte Carlo Methods (H. A. Meyer, editor), 146-190. New York: John Wiley \& Sons. Use of different Monte Carlo sampling techniques.

29. B. M. AyYub and R. H. MCCuen 1997 in Probabilistic Structural Mechanics Handbook: Theory and Industrial Applications (C. Sundarajan, editor), 53-69. New York: Chapman \& Hall. Simulation based reliability methods.

30. G. I. SCHUELLER and R. STIX 1987 Structural Safety 4, 293-309. A critical appraisal of methods to determine failure probabilities.

31. C. G. Bucher 1988 Structural Safety 5, 119-126. Adaptive sampling - an iterative fast Monte Carlo procedure.

32. A. Karamchandani, P. BJerager and C. A. Cornell 1989 in Proceedings of the 5th International Conference on Structural Safety and Reliability (ICOSSAR'89), (A. H-S. Ang, M. Shinozuka and G. I. Schueller, editors), 855-862. New York: ASCE. Adaptive importance sampling.

\section{APPENDIX A: FORMULATION OF SHAPE FUNCTIONS FOR DISPLACEMENTS}

For the beam element with homogenous properties, equations (20-22) can be recast as

$$
\begin{gathered}
\alpha_{1} \frac{\mathrm{d}^{2} w}{\mathrm{~d} \phi^{2}}+\alpha_{2} w+\alpha_{3} \frac{\mathrm{d} w}{\mathrm{~d} \phi}+\alpha_{4} \frac{\mathrm{d} \psi}{\mathrm{d} \phi}=0, \\
\beta_{1} \frac{\mathrm{d}^{2} v}{\mathrm{~d} \phi^{2}}+\beta_{2} v+\beta_{3} \frac{\mathrm{d} w}{\mathrm{~d} \phi}+\beta_{4} \psi=0, \\
\gamma_{1} \frac{\mathrm{d}^{2} \psi}{\mathrm{d} \phi^{2}}+\gamma_{2} \psi+\gamma_{3} \frac{\mathrm{d} \psi}{\mathrm{d} \phi}+\gamma_{4} v=0,
\end{gathered}
$$

where

$$
\begin{gathered}
\alpha_{1}=\frac{\bar{k} A G}{R}, \quad \beta_{1}=\frac{E A}{R}, \quad \gamma_{1}=\frac{E J}{R}, \\
\alpha_{2}=\omega^{2} \rho A R-\frac{E A}{R}-\mathrm{i} \omega c_{1}, \quad \beta_{2}=-\frac{\bar{k} A G}{R}+\omega^{2} \rho A R-\mathrm{i} \omega c_{2}, \\
\gamma_{2}=\omega^{2} \rho J R-\bar{k} A G R-\mathrm{i} \omega c_{3}, \\
\alpha_{3}=\frac{E A+\bar{k} A G}{R}, \quad \beta_{3}=-\frac{E A+\bar{k} G A}{R}, \quad \gamma_{3}=\bar{k} A G, \\
\alpha_{4}=-\bar{k} A G, \quad \beta_{4}=\bar{k} A G, \quad \gamma_{4}=\bar{k} A G .
\end{gathered}
$$


From equation (A.2), $\psi$ can be expressed as

$$
\psi=-\frac{1}{\beta_{4}}\left\{\beta_{1} \frac{\mathrm{d}^{2} v}{\mathrm{~d} \phi^{2}}+\beta_{2} v+\beta_{3} \frac{\mathrm{d} w}{\mathrm{~d} \phi}\right\} .
$$

Consequently, the first and second derivatives of $\psi$ with respect to $\phi$ are obtained by successive differentiations of equation (A.4). Substituting for $\psi$ into equations (A.1) and (A.3), $\psi$ is eliminated and two coupled differential equations in $w$ and $v$ are obtained:

$$
L_{1} w+L_{2} v=0, \quad L_{3} w+L_{4} v=0
$$

where $L_{i}(i=1, \ldots, 4)$ are operators denoted by

$$
\begin{array}{cc}
L_{1}=A_{1} \frac{\mathrm{d}^{2}}{\mathrm{~d} \phi^{2}}+A_{2}, & L_{2}=A_{3} \frac{\mathrm{d}^{3}}{\mathrm{~d} \phi^{3}}+A_{4} \frac{\mathrm{d}}{\mathrm{d} \phi} \\
L_{3}=B_{4} \frac{\mathrm{d}^{3}}{\mathrm{~d} \phi^{3}}+B_{5} \frac{\mathrm{d}}{\mathrm{d} \phi}, & L_{4}=B_{1} \frac{\mathrm{d}^{4}}{\mathrm{~d} \phi^{4}}+B_{2} \frac{\mathrm{d}^{2}}{\mathrm{~d} \phi^{2}}+B_{3}
\end{array}
$$

with

$$
\begin{array}{ll}
A_{1}=\alpha_{1}-\alpha_{4} \beta_{3} / \beta_{4}, & B_{1}=-\gamma_{1} \beta_{1} / \beta_{4}, \\
A_{2}=\alpha_{2}, & B_{2}=-\left(\gamma_{2} \beta_{1}+\gamma_{1} \beta_{2}\right) / \beta_{4}, \\
A_{3}=-\alpha_{4} \beta_{1} / \beta_{4}, & B_{3}=\gamma_{4}-\gamma_{2} \beta_{2} / \beta_{4}, \\
A_{4}=\alpha_{3}-\alpha_{4} \beta_{2} / \beta_{4}, & B_{4}=-\gamma_{1} \beta_{3} / \beta_{4}, \\
B_{5}=\gamma_{3}-\gamma_{2} \beta_{3} / \beta_{4}, &
\end{array}
$$

Equations (A.5-A.6) are now combined to obtain

$$
\begin{aligned}
& \left(L_{1} L_{4}-L_{2} L_{3}\right) v=0, \\
& \left(L_{1} L_{4}-L_{2} L_{3}\right) w=0 .
\end{aligned}
$$

Expanding equations (A.8-A.9), sixth order homogeneous ordinary differential equations in $w$ and $v$ are obtained as follows:

$$
\begin{aligned}
& \left(K_{1} \frac{\mathrm{d}^{6}}{\mathrm{~d} \phi^{6}}+K_{2} \frac{\mathrm{d}^{4}}{\mathrm{~d} \phi^{4}}+K_{3} \frac{\mathrm{d}^{2}}{\mathrm{~d} \phi^{2}}+K_{4}\right) w=0, \\
& \left(K_{1} \frac{\mathrm{d}^{6}}{\mathrm{~d} \phi^{6}}+K_{2} \frac{\mathrm{d}^{4}}{\mathrm{~d} \phi^{4}}+K_{3} \frac{\mathrm{d}^{2}}{\mathrm{~d} \phi^{2}}+K_{4}\right) v=0 .
\end{aligned}
$$

Here,

$$
\begin{gathered}
K_{1}=A_{1} B_{1}-A_{3} B_{4}, \quad K_{2}=A_{1} B_{2}+A_{2} B_{1}-A_{3} B_{5}-A_{4} B_{4}, \\
K_{3}=A_{1} B_{3}+A_{2} B_{2}-A_{4} B_{5}, \quad K_{4}=A_{2} B_{3} .
\end{gathered}
$$


Thus, it may be noted that equations for $w$ and $v$ are now mutually uncoupled. It is also of interest to note that the differential operators which act on $w$ and $v$ in equations (A.10) and (A.11) are identical.

Assuming the solution of equation (A.10) to be of the form $w=\exp [\mu \phi]$, characteristic equation for $\mu$ is obtained as

$$
\mu^{6} K_{1}+\mu^{4} K_{2}+\mu^{2} K_{3}+K_{4}=0 .
$$

An identical characteristic equation would also be obtained if a solution of the form $v=\exp [\mu \phi]$ is substituted into equation (A.11). Furthermore, the six roots of equation (A.13), using symbolic math processing (MAPLE), are obtained as:

$$
\begin{aligned}
& \mu_{1}=\frac{1}{3}\left(9 v^{1 / 3}-\frac{3 K_{3} K_{1}-K_{2}^{2}}{K_{1}^{2} v^{1 / 3}}-3 \frac{K_{2}}{K_{1}}\right)^{1 / 2} \\
& \mu_{2}=-\frac{1}{3}\left(9 v^{1 / 3}-\frac{3 K_{3} K_{1}-K_{2}^{2}}{K_{1}^{2} v^{1 / 3}}-3 \frac{K_{2}}{K_{1}}\right)^{1 / 2} \\
& \mu_{3}=\frac{1}{6}\left(-18 v^{1 / 3}+\left(\frac{3 K_{3} K_{1}-K_{2}^{2}}{K_{1}^{2} v^{1 / 3}}\right)-12 \frac{K_{2}}{K_{1}}+18 \lambda\right)^{1 / 2}, \\
& \mu_{4}=-\frac{1}{6}\left(-18 v^{1 / 3}+\left(\frac{3 K_{3} K_{1}-K_{2}^{2}}{K_{1}^{2} v^{1 / 3}}\right)-12 \frac{K_{2}}{K_{1}}+18 \lambda\right)^{1 / 2}, \\
& \mu_{5}=\frac{1}{6}\left(-18 v^{1 / 3}+\left(\frac{3 K_{3} K_{1}-K_{2}^{2}}{K_{1}^{2} v^{1 / 3}}\right)-12 \frac{K_{2}}{K_{1}}-18 \lambda\right)^{1 / 2}, \\
& \mu_{6}=-\frac{1}{6}\left(-18 v^{1 / 3}+\left(\frac{3 K_{3} K_{1}-K_{2}^{2}}{K_{1}^{2} v^{1 / 3}}\right)-12 \frac{K_{2}}{K_{1}}-18 \lambda\right)^{1 / 2},
\end{aligned}
$$

where

$$
\begin{gathered}
v=-\frac{1}{54}\left(\frac{-9 K_{3} K_{2} K_{1}+27 K_{4} K_{1}^{2}+2 K_{2}^{3}}{K_{1}^{3}}\right) \\
\times \frac{\sqrt{3}}{18 K_{1}^{2}}\left(4 K_{3}^{3} K_{1}-K_{3}^{2} K_{2}^{2}-18 K_{3} K_{2} K_{1} K_{4}+27 K_{4}^{2} K_{1}^{2}+4 K_{4} K_{2}^{3}\right)^{1 / 2} \\
\lambda=\mathrm{i} \sqrt{3}\left\{v^{1 / 3}+\frac{1}{9}\left(\frac{3 K_{3} K_{1}-K_{2}^{2}}{K_{1}^{2} v^{1 / 3}}\right)\right\} .
\end{gathered}
$$

Thus, the displacements $v$ and $w$ can now be expressed as summations of series as follows:

$$
w(\phi)=\sum_{k=1}^{6} g_{k} \exp \left[\mu_{k} \phi\right], \quad v(\phi)=\sum_{k=1}^{6} f_{k} \exp \left[\mu_{k} \phi\right] .
$$


Here $g_{k}$ and $f_{k}$ are constants of integration to be determined from the boundary conditions. At this stage, it is seen that there are twelve integration constants that need to be determined. These constants are however not mutually independent. Substituting equations (A.17) and (A.18) into equation (A.8), it is possible to obtain the following relationship between the constants of integration $g_{k}$ and $f_{k}$ :

$$
f_{k}=\zeta_{k} g_{k}
$$

where

$$
\zeta_{k}=\frac{A_{1} \mu_{k}^{2}+A_{2}}{A_{3} \mu_{k}^{3}+A_{4} \mu_{k}} .
$$

Substituting the expressions for the displacements $w$ and $v$ into equation (A.4), the displacement $\psi$ is expressed as

$$
\psi(\phi)=\sum_{k=1}^{6} \xi_{k} g_{k} \exp \left[\mu_{k} \phi\right]
$$

where

$$
\xi_{k}=\left(-\frac{\beta_{1}}{\beta_{4}} \mu_{k}^{2}-\frac{\beta_{2}}{\beta_{4}}\right) \zeta_{k}-\frac{\beta_{3}}{\beta_{4}} \mu_{k} .
$$

Thus, all the three displacements $w, v$ and $\psi$ can be represented in a series form. It must be noted that this involves only six independent unknown constants of integration which can be determined from the six boundary conditions. Thus, the solution of the problem is exactly determinable.

The shape functions $N_{k}(\phi, \omega), P_{k}(\phi, \omega)$ and $Q_{k}(\phi, \omega)$ in equations (25-27) are derived by solving the field equations (20-22) in conjunction with the appropriate boundary conditions. Table A1 lists the boundary conditions satisfied by the three sets of shape functions. Writing the binary conditions in matrix form,

$$
\left.[\Gamma]\{\mathbf{g}\}^{1},\{\mathbf{g}\}^{2},\{\mathbf{g}\}^{3},\{\mathbf{g}\}^{4},\{\mathbf{g}\}^{5},\{\mathbf{g}\}^{6}\right]=\mathbf{I},
$$

\section{TABLE A1}

Relationship between "binary" boundary conditions and shape functions $N_{n}(\phi, \Omega), P_{n}(\phi, \Omega)$ and $Q_{n}(\phi, \Omega)$

\begin{tabular}{ccccccc}
\hline & $N_{1}(\phi, \omega)$, & $N_{2}(\phi, \omega)$, & $N_{3}(\phi, \omega)$, & $N_{4}(\phi, \omega)$, & $N_{5}(\phi, \omega)$, & $N_{6}(\phi, \omega)$, \\
& $P_{1}(\phi, \omega)$, & $P_{2}(\phi, \omega)$, & $P_{3}(\phi, \omega)$, & $P_{4}(\phi, \omega)$, & $P_{5}(\phi, \omega)$, & $P_{6}(\phi, \omega)$, \\
& $Q_{1}(\phi, \omega)$ & $Q_{2}(\phi, \omega)$ & $Q_{3}(\phi, \omega)$ & $Q_{4}(\phi, \omega)$ & $Q_{5}(\phi, \omega)$ & $Q_{6}(\phi, \omega)$ \\
\hline$w(0)$ & 1 & 0 & 0 & 0 & 0 & 0 \\
$v(0)$ & 0 & 1 & 0 & 0 & 0 & 0 \\
$\psi(0)$ & 0 & 0 & 1 & 0 & 0 & 0 \\
$w(L)$ & 0 & 0 & 0 & 1 & 0 & 0 \\
$v(L)$ & 0 & 0 & 0 & 0 & 1 & 0 \\
$\psi(L)$ & 0 & 0 & 0 & 0 & 0 & 1 \\
\hline
\end{tabular}


where

$$
[\Gamma(\omega)]=\left[\begin{array}{llllll}
\mathrm{e}^{\mu_{1} \phi_{0}} & \mathrm{e}^{\mu_{2} \phi_{0}} & \mathrm{e}^{\mu_{3} \phi_{0}} & \mathrm{e}^{\mu_{4} \phi_{0}} & \mathrm{e}^{\mu_{5} \phi_{0}} & \mathrm{e}^{\mu_{6} \phi_{0}} \\
\zeta_{1} \mathrm{e}^{\mu_{1} \phi_{0}} & \zeta_{2} \mathrm{e}^{\mu_{2} \phi_{0}} & \zeta_{3} \mathrm{e}^{\mu_{3} \phi_{0}} & \zeta_{4} \mathrm{e}^{\mu_{4} \phi_{0}} & \zeta_{5} \mathrm{e}^{\mu_{5} \phi_{0}} & \zeta_{6} \mathrm{e}^{\mu_{6} \phi_{0}} \\
\xi_{1} \mathrm{e}^{\mu_{1} \phi_{0}} & \xi_{2} \mathrm{e}^{\mu_{2} \phi_{0}} & \xi_{3} \mathrm{e}^{\mu_{3} \phi_{0}} & \xi_{4} \mathrm{e}^{\mu_{4} \phi_{0}} & \xi_{5} \mathrm{e}^{\mu_{5} \phi_{0}} & \xi_{6} \mathrm{e}^{\mu_{6} \phi_{0}} \\
\mathrm{e}^{\mu_{1} \phi_{f}} & \mathrm{e}^{\mu_{2} \phi_{f}} & \mathrm{e}^{\mu_{3} \phi_{f}} & \mathrm{e}^{\mu_{4} \phi_{f}} & \mathrm{e}^{\mu_{5} \phi_{f}} & \mathrm{e}^{\mu_{6} \phi_{f}} \\
\zeta_{1} \mathrm{e}^{\mu_{1} \phi_{f}} & \zeta_{2} \mathrm{e}^{\mu_{2} \phi_{f}} & \zeta_{3} \mathrm{e}^{\mu_{3} \phi_{f}} & \zeta_{4} \mathrm{e}^{\mu_{4} \phi_{f}} & \zeta_{5} \mathrm{e}^{\mu_{5} \phi_{f}} & \zeta_{6} \mathrm{e}^{\mu_{6} \phi_{f}} \\
\xi_{1} \mathrm{e}^{\mu_{1} \phi_{f}} & \xi_{2} \mathrm{e}^{\mu_{2} \phi_{f}} & \xi_{3} \mathrm{e}^{\mu_{3} \phi_{f}} & \xi_{4} \mathrm{e}^{\mu_{4} \phi_{f}} & \xi_{5} \mathrm{e}^{\mu_{5} \phi_{f}} & \xi_{6} \mathrm{e}^{\mu_{6} \phi_{f}}
\end{array}\right]
$$

and $\left\{\mathbf{g}^{k}\right\}$ is the vector of constants giving rise to the $k$ th shape function. To proceed further, the shape functions $N_{k}(\phi, \omega), P_{k}(\phi, \omega)$ and $Q_{k}(\phi, \omega)$ are represented in matrix form, as in equations (A.25-A.27). Combining equations (A.14) with the requirements of the boundary conditions listed in Table A1, it can be shown that

$$
\begin{aligned}
& \mathbf{N}(\phi, \omega)=[\boldsymbol{\Gamma}(\omega)]\left\{\mathbf{s}_{1}(\phi, \omega)\right\}, \\
& \mathbf{P}(\phi, \omega)=[\boldsymbol{\Gamma}(\omega)]\left\{\mathbf{s}_{2}(\phi, \omega)\right\}, \\
& \mathbf{Q}(\phi, \omega)=[\boldsymbol{\Gamma}(\omega)]\left\{\mathbf{s}_{3}(\phi, \omega)\right\},
\end{aligned}
$$

where

$$
\begin{gathered}
\Gamma(\phi, \omega)=\left[\{\mathbf{g}\}^{1},\{\mathbf{g}\}^{2},\{\mathbf{g}\}^{3},\{\mathbf{g}\}^{4},\{\mathbf{g}\}^{5},\{\mathbf{g}\}^{6}\right]^{\mathrm{t}}, \\
\left\{\mathbf{s}_{1}\right\}=\left[\mathbf{e}^{\mu_{1} \phi}, \mathbf{e}^{\mu_{2} \phi}, \mathbf{e}^{\mu_{3} \phi}, \mathbf{e}^{\mu_{4} \phi}, \mathbf{e}^{\mu_{5} \phi}, \mathbf{e}^{\mu_{6} \phi}\right], \\
\left\{\mathbf{s}_{2}\right\}=\left[\zeta_{1} \mathbf{e}^{\mu_{1} \phi}, \zeta_{2} \mathbf{e}^{\mu_{2} \phi}, \zeta_{3} \mathbf{e}^{\mu_{3} \phi}, \zeta_{4} \mathbf{e}^{\mu_{4} \phi}, \zeta_{5} \mathbf{e}^{\mu_{5} \phi}, \zeta_{6} \mathbf{e}^{\mu_{6} \phi}\right], \\
\left\{\mathbf{s}_{3}\right\}=\left[\xi_{1} \mathbf{e}^{\mu_{1} \phi}, \xi_{2} \mathbf{e}^{\mu_{2} \phi}, \xi_{3} \mathbf{e}^{\mu_{3} \phi}, \xi_{4} \mathbf{e}^{\mu_{4} \phi}, \xi_{5} \mathbf{e}^{\mu_{5} \phi}, \xi_{6} \mathbf{e}^{\mu_{6} \phi}\right]
\end{gathered}
$$

The shape functions derived in this manner are functions of frequency and are complex valued. The formulations for the circular beam are expected to lead to the corresponding results for a straight Timoshenko beam as the radius $R \rightarrow \infty$. However, it is simpler to reformulate the field equations for a straight Timoshenko beam separately. Further details of this formulation are available in reference [20]. 